\title{
On New Modifications Governed by Quantum Hahn's Integral Operator Pertaining to Fractional Calculus
}

\author{
Saima Rashid $\mathbb{D},{ }^{1}$ Aasma Khalid, ${ }^{2}$ Gauhar Rahman $\mathbb{D}^{3},{ }^{3}$ Kottakkaran Sooppy Nisar, ${ }^{4}$ \\ and Yu-Ming Chu $\oplus^{5,6}$ \\ ${ }^{1}$ Department of Mathematics, Government College University, Faisalabad 38000, Pakistan \\ ${ }^{2}$ Department of Mathematics, Government College Women University Faisalabad, Madina Town, Faisalabad 38000, Pakistan \\ ${ }^{3}$ Department of Mathematics, Shaheed Benazir Bhutto University, Sheringal, Pakistan \\ ${ }^{4}$ Department of Mathematics, College of Arts and Sciences, Prince Sattam Bin Abdulaziz University, \\ Wadi Al Dawasir 11991, Saudi Arabia \\ ${ }^{5}$ Department of Mathematics, Huzhou University, Huzhou 313000, China \\ ${ }^{6}$ Hunan Provincial Key Laboratory of Mathematical Modeling and Analysis in Engineering, Changsha University of Science \\ \& Technology, Changsha 410114, China
}

Correspondence should be addressed to Yu-Ming Chu; chuyuming@zjhu.edu.cn

Received 17 April 2020; Accepted 23 June 2020; Published 14 July 2020

Academic Editor: Gestur Ólafsson

Copyright (c) 2020 Saima Rashid et al. This is an open access article distributed under the Creative Commons Attribution License, which permits unrestricted use, distribution, and reproduction in any medium, provided the original work is properly cited.

In the article, we present several generalizations for the generalized Čebyšev type inequality in the frame of quantum fractional Hahn's integral operator by using the quantum shift operator ${ }_{\sigma} \Psi_{\mathfrak{q}}(\varsigma)=\mathfrak{q} \varsigma+(1-\mathfrak{q}) \sigma\left(\varsigma \in\left[l_{1}, l_{2}\right], \sigma=l_{1}+\omega /(1-\mathfrak{q}), 0<\mathfrak{q}<1, \omega \geq 0\right)$. As applications, we provide some associated variants to illustrate the efficiency of quantum Hahn's integral operator and compare our obtained results and proposed technique with the previously known results and existing technique. Our ideas and approaches may lead to new directions in fractional quantum calculus theory.

\section{Introduction}

In 1882, Čebyšev discovered a fascinating and significantly valuable integral inequality as follows:

$$
\begin{aligned}
& \frac{1}{l_{1}-l_{2}} \int_{l_{1}}^{l_{2}} \mathscr{Q}(x) \mathscr{U}(x) d x \\
& \quad \leq\left(\frac{1}{l_{1}-l_{2}} \int_{l_{1}}^{l_{2}} \mathscr{Q}(x) d x\right)\left(\frac{1}{l_{1}-l_{2}} \int_{l_{1}}^{l_{2}} \mathcal{U}(x) d x\right),
\end{aligned}
$$

if $\mathbb{Q}$ and $\mathcal{U}$ are two integrable and synchronous functions on $\left[l_{1}, l_{2}\right]$, where the functions $\mathcal{Q}$ and $\mathcal{U}$ are said to be synchronous on $\left[l_{1}, l_{2}\right]$ if

$$
(\mathscr{Q}(x)-\mathscr{Q}(y))(\mathcal{U}(x)-\mathscr{U}(y)) \geq 0
$$

for all $x, y \in\left[l_{1}, l_{2}\right]$.
It is well known that the Čebyšev inequality (1) has wild applications in the fields of pure and applied mathematics [1-10]. Recently, the generalizations and variants for the Čebyšev inequality (1) have attracted the attention of many researchers [11-20].

Quantum difference operators are receiving an increase of interest due to their applications [21, 22]. Roughly speaking, quantum calculus can substitute the classical derivative by a difference operator, which allows to deal nondifferentiation functions.

Let $\mathfrak{q} \in(0,1), \omega \geq 0, I \subseteq \mathbb{R}$ be an interval such that $\omega_{0}=$ $\omega /(1-\mathfrak{q}) \in I$, and $\mathscr{H}_{1}: I \longrightarrow \mathbb{R}$ be a real-valued function. Then, the Hahn difference operator $\mathscr{D}_{\mathfrak{q}, \omega}$ [23] is defined by

$$
\mathscr{D}_{\mathfrak{q}, \omega} \mathscr{H}_{1}(\varsigma)= \begin{cases}\frac{\mathscr{H}_{1}(\mathfrak{q} \varsigma+\omega)-\mathscr{H}_{1}(\varsigma)}{\varsigma(\mathfrak{q}-1)+\omega}, & \varsigma \neq \omega_{0}, \\ \mathscr{H}_{1}^{\prime}\left(\omega_{0}\right), & \varsigma=\omega_{0},\end{cases}
$$

if $\mathscr{H}_{1}$ is differentiable at $\omega_{0}$. 
The Hahn difference operator (3) unifies (in the limit) the Jackson $q$-difference derivative $\mathscr{D}_{\mathfrak{q}}[24]$ for $\mathfrak{q} \in(0,1)$ and the forward difference $\mathscr{D}_{\omega}$ for $\mathfrak{q} \longrightarrow 1$, which are defined by

$$
\mathscr{D}_{\mathfrak{q}} \mathscr{H}_{1}(\varsigma)= \begin{cases}\frac{\mathscr{H}_{1}(\varsigma)-\mathscr{H}_{1}(\mathfrak{q} \varsigma)}{\varsigma(1-\mathfrak{q})}, & \varsigma \neq 0, \\ \mathscr{H}_{1}^{\prime}(0), & \varsigma=0,\end{cases}
$$

if $\mathscr{H}_{1}^{\prime}(0)$ exists for $\omega=0$, and

$$
\mathscr{D}_{\omega} \mathscr{H}_{1}(\varsigma)=\frac{\mathscr{H}_{1}(\varsigma+\omega)-\mathscr{H}_{1}(\varsigma)}{\omega}
$$

for $\omega>0$.

The Hahn difference operator has been applied successfully in the construction of families of orthogonal polynomials as well as in approximation problems [25-28].

In [29], the authors introduced some concepts of fractional quantum calculus in terms of a $\mathfrak{q}$-shifting operator ${ }_{\sigma} \Psi_{\mathfrak{q}}(\varsigma)=\mathfrak{q} \varsigma+(1-\mathfrak{q}) \sigma$.

Let $I=\left[l_{1}, l_{2}\right] \subseteq \mathbb{R}$ be an interval. Then, the point $\sigma$ of Hahn calculus on the interval $\left[l_{1}, l_{2}\right]$ generated by the quantum numbers $0<\mathfrak{q}<1$ and $\omega \geq 0$ is given by

$$
\sigma=l_{1}+\frac{\omega}{1-\mathfrak{q}}
$$

We state that $\sigma \in\left[l_{1}, l_{2}\right]$ for all consequences of our investigation; the quantum Hahn shifting operator is defined by

$$
{ }_{\sigma} \Psi_{\mathfrak{q}}(\varsigma)=\mathfrak{q} \varsigma+(1-\mathfrak{q}) \sigma, \quad \varsigma \in\left[l_{1}, l_{2}\right]
$$

and the iterated $\kappa$-times quantum shifting is given by

$$
{ }_{\sigma} \Psi_{\mathfrak{q}}^{\kappa}(\varsigma)={ }_{\sigma} \Psi_{\mathfrak{q}}^{\kappa-1}\left({ }_{\sigma} \Psi_{\mathfrak{q}}(\varsigma)\right)=\mathfrak{q}^{\kappa} \varsigma+\left(1-\mathfrak{q}^{\kappa}\right) \sigma
$$

with ${ }_{\sigma} \Psi_{\mathfrak{q}}^{0}(\varsigma)=\varsigma$ for $\varsigma \in\left[l_{1}, l_{2}\right]$.

Let us recall the basic knowledge of quantum Hahn calculus on an interval $\left[l_{1}, l_{2}\right]$ (see [30]).

Definition 1 . Let $\mathscr{H}_{1}$ be a function defined on $\left[l_{1}, l_{2}\right]$. Then, the quantum Hahn difference operator is defined by

$$
l_{1} \mathscr{D}_{\mathfrak{q}, \omega} \mathscr{H}_{1}(\varsigma)= \begin{cases}\frac{\mathscr{H}_{1}(\varsigma)-\mathscr{H}_{1}\left({ }_{\sigma} \Psi_{\mathfrak{q}}(\varsigma)\right)}{\varsigma-{ }_{\sigma} \Psi_{\mathfrak{q}}(\varsigma)}, & \varsigma \neq \sigma, \\ \mathscr{H}_{1}^{\prime}(\sigma), & \varsigma=\sigma,\end{cases}
$$

if $\mathscr{H}_{1}$ is differentiable at $\sigma$.

Definition 2. Let $\mathscr{H}_{1}:\left[l_{1}, l_{2}\right] \longrightarrow \mathbb{R}$ be a given function and $x, y \in\left[l_{1}, l_{2}\right]$. Then, the $\mathfrak{q}, \omega$-quantum Hahn integral of $\mathscr{H}_{1}$ from $x$ to $y$ is defined by

$$
\int_{x}^{y} \mathscr{H}_{1}(s)_{l_{1}} d_{\mathfrak{q}, \omega} s=\int_{\sigma}^{y} \mathscr{H}_{1}(s)_{l_{1}} d_{\mathfrak{q}, \omega} s-\int_{\sigma}^{x} \mathscr{H}_{1}(s)_{l_{1}} d_{\mathfrak{q}, \omega} s,
$$

where

$$
\int_{\sigma}^{\varsigma} \mathscr{H}_{1}(s)_{l_{1}} d_{\mathfrak{q}, \omega} s=\left[\varsigma-{ }_{\sigma} \Psi_{\mathfrak{q}}(\varsigma)\right] \sum_{i=0}^{\infty} \mathfrak{q}^{i} \mathscr{H}_{1}\left({ }_{\sigma} \Psi_{\mathfrak{q}}^{i}(\varsigma)\right)
$$

for $\varsigma \in\left[l_{1}, l_{2}\right]$ provided that the series converge at $\varsigma=x$ and $\varsigma=y$. The function $\mathscr{H}_{1}$ is said to be $\mathfrak{q}, \omega$-integrable on $\left[l_{1}, l_{2}\right]$ if (11) exists for all $\varsigma \in\left[l_{1}, l_{2}\right]$.

Before approaching the main definitions of fractional quantum Hahn calculus on $\left[l_{1}, l_{2}\right]$, we present the $\sigma$-power function which is stated as

$$
\begin{aligned}
(n-m)_{\sigma}^{(0)} & =1, \\
(n-m)_{\sigma}^{(\kappa)} & =\prod_{i=0}^{\kappa-1}\left(n-{ }_{\sigma} \Psi_{\mathfrak{q}}^{i}(m)\right), \\
\kappa & \in \mathbb{N} \cup\{\infty\} .
\end{aligned}
$$

Precisely, if $\alpha \in \mathbb{R}$, then

$$
(n-m)_{\sigma}^{(\alpha)}=\prod_{i=0}^{\infty} \frac{\left(n-{ }_{\sigma} \Psi_{\mathfrak{q}}^{i}(m)\right)}{\left(n-{ }_{\sigma} \Psi_{\mathfrak{q}}^{\alpha+i}(m)\right)},
$$

with ${ }_{\sigma} \Psi_{\mathfrak{q}}^{\varsigma}(m)=\mathfrak{q}^{\varsigma} m+\left(1-\mathfrak{q}^{\varsigma}\right) \sigma$ for $\varsigma \in \mathbb{R}$.

The $\mathfrak{q}$-gamma function is defined as

$$
\Gamma_{\mathfrak{q}}(\eta)=\frac{(1-\mathfrak{q})_{0}^{\eta-1}}{(1-\mathfrak{q})^{\eta-1}}
$$

for $\eta \in \mathbb{R} \backslash\{0,-1,-2, \cdots\}$.

Obviously, $\Gamma_{\mathfrak{q}}(\eta+1)=[\alpha]_{\mathfrak{q}} \Gamma_{\mathfrak{q}}(\eta)$, where $[c]_{\mathfrak{q}}=\left(1-\mathfrak{q}^{c}\right) /$ $(1-\mathfrak{q})(c \in \mathbb{R})$ and $\mathfrak{q}$ is the quantum number.

Now, we introduce the concepts of fractional quantum Hahn derivative and integral of Riemann-Liouville type [31].

Definition 3 (see [31]). Suppose that $\alpha \geq 0$ and $\mathscr{H}_{1}:\left[l_{1}, l_{2}\right]$ $\longrightarrow \mathbb{R}$ is a real-valued function. Then, the fractional quantum Hahn derivative $\left({ }_{l_{1}} \mathscr{D}_{\mathfrak{q}, \omega}^{\alpha} \mathscr{H}_{1}\right)(\varsigma)$ of the Riemann-Liouville type of order $\alpha$ is defined by

$$
\begin{aligned}
& \left(l_{1} \mathscr{D}_{\mathfrak{q}, \omega}^{\alpha} \mathscr{H}_{1}\right)(\varsigma) \\
& \quad=\frac{1}{\Gamma_{\mathfrak{q}}(n-\alpha)_{l_{1}}} \mathscr{D}_{\mathfrak{q}, \omega}^{n} \int_{l_{1}}^{\varsigma}\left(\varsigma-{ }_{\sigma} \Psi_{\mathfrak{q}}(s)\right)_{\sigma}^{n-\alpha-1} \mathscr{H}_{1}(s)_{l_{1}} d_{\mathfrak{q}, \omega},
\end{aligned}
$$

where $n$ is the smallest integer greater than or equal to $\alpha$.

Definition 4 (see [31]). Let $\alpha \geq 0$ and $\mathscr{H}_{1}:\left[l_{1}, l_{2}\right] \longrightarrow \mathbb{R}$ be a real-valued function. Then, the fractional quantum Hahn 
integral $\left(l_{1} \mathscr{J}_{\mathfrak{q}, \omega}^{\alpha} \mathscr{H}_{1}\right)(\varsigma)$ of the Riemann-Liouville type of order $\alpha$ is defined by

$$
\begin{aligned}
& \left(l_{1} \mathscr{J}_{\mathfrak{q}, \omega}^{\alpha} \mathscr{H}_{1}\right)(\varsigma) \\
& \quad=\frac{1}{\Gamma_{\mathfrak{q}}(\alpha)} \int_{l_{1}}^{\varsigma}\left(\varsigma-{ }_{\sigma} \Psi_{\mathfrak{q}}(s)\right)_{\sigma}^{\alpha-1} \mathscr{H}_{1}(s)_{l_{1}} d_{\mathfrak{q}, \omega}, \quad \varsigma \in\left[l_{1}, l_{2}\right]
\end{aligned}
$$

with $\left({ }_{l} \mathscr{J}_{\mathfrak{q}, \omega}^{l_{1}} \mathscr{H}_{1}\right)(\varsigma)=\mathscr{H}_{1}(\varsigma)$ if the right-hand side exists.

Theorem 5 (see [31]). Let $\alpha, \beta \in \mathbb{R}^{+}, \vartheta \in(-1, \infty)$, and $\sigma \in$ $\left[l_{1}, l_{2}\right]$. Then, one has

$$
\begin{aligned}
& \left(l_{1} \mathscr{I}_{\mathfrak{q}, \omega}^{\alpha}\left(x-l_{1}\right)_{\sigma}^{\vartheta}\right)(\varsigma)=\frac{\Gamma_{\mathfrak{q}}(\vartheta+1)}{\Gamma_{\mathfrak{q}}(\alpha+\vartheta+1)}\left(\varsigma-l_{1}\right)_{\sigma}^{\alpha+\vartheta} \\
& \left(l_{1} \mathscr{D}_{\mathfrak{q}, \omega}^{\alpha}\left(x-l_{1}\right)_{\sigma}^{\vartheta}\right)(\varsigma)=\frac{\Gamma_{\mathfrak{q}}(\vartheta+1)}{\Gamma_{\mathfrak{q}}(\alpha-\vartheta+1)}\left(\varsigma-l_{1}\right)_{\sigma}^{\alpha-\vartheta}
\end{aligned}
$$

Fractional calculus is invariably important in almost all fields of mathematics and applied sciences. Also, the fractional differential equations can provide adequate models for many physical problems in areas such as heat equation, wave equation, Poisson equation and Laplace equation, fluid mechanics, biological populations, viscoelasticity, advectiondiffusion, and signal processing $[32,33]$.

Inequality plays an irreplaceable role in the development of mathematics. Very recently, many new inequalities such as Hermite-Hadamard type inequality [34-38], Petrović type inequality [39], Pólya-Szegö type inequality [40], Ostrowski type inequality [41], reverse Minkowski inequality [42], Jensen type inequality $[43,44]$, Bessel function inequality [45], trigonometric and hyperbolic function inequalities [46], fractional integral inequality [47-51], complete and generalized elliptic integral inequalities [52-57], generalized convex function inequality [58-60], and mean value inequality [61-63] have been discovered by many researchers. In particular, the applications of integral inequalities have gained considerable importance among researchers for fixed-point theorems; the existence and uniqueness of solutions for differential equations [64-68] and numerous numerical and analytical methods have been recommended for the advancement of integral inequalities [69-75].

Asawasamrit et al. [76] expounded the concept of $\mathfrak{q}$ derivative over the interval $\left[l_{1}, l_{2}\right] \subset \mathbb{R}$ and derived several inequalities on quantum analogues, for example, $\mathfrak{q}$-CauchySchwarz inequality, $\mathfrak{q}$-Grüss-Čebyšev integral inequality, $\mathfrak{q}$ Grüss inequality, and other integral inequalities, by use of the convexity theory.

The main purpose of the article is to provide the novel versions of the generalized Čebyšev inequalities and present the associated variants via quantum Hahn's fractional integral operator.

To end this section, we give the definition of the onesided fractional quantum Hahn integral in the RiemannLiouville sense.
Definition 6. Let $\alpha \geq 0$ and $\mathscr{H}_{1}:\left[l_{1}, l_{2}\right] \longrightarrow \mathbb{R}$ be a real-valued function. Then, the one-sided fractional quantum Hahn integral $\left({ }_{0^{+}} \mathscr{J}_{\mathbf{q}, \omega}^{\alpha} \mathscr{H}_{1}\right)(\varsigma)$ of Riemann-Liouville type of order $\alpha$ is defined by

$$
\left(0_{0^{+}} \mathscr{J}_{\mathfrak{q}, \omega}^{\alpha} \mathscr{H}_{1}\right)(\varsigma)=\frac{1}{\Gamma_{\mathfrak{q}}(\alpha)} \int_{0}^{\varsigma}\left(\varsigma^{-}{ }_{\sigma} \Psi_{\mathfrak{q}}(s)\right)_{\sigma}^{\alpha-1} \mathscr{H}_{1}(s)_{l_{1}} d_{\mathfrak{q}, \omega}, \quad s>0
$$

\section{Certain Extended Weighted Čebyšev Fractional Quantum Hahn Integral Operator}

In this section, we provide several new generalizations for the weighted extensions of Čebyšev functionals via a quantum Hahn integral operator.

Theorem 7. Let $s, s_{1}, u, u_{1}, r, r_{1}>1$ with $s^{-1}+s_{1}^{-1}=r^{-1}+$ $r_{1}^{-1}=u^{-1}+u_{1}^{-1}=1, \mathfrak{q} \in(0,1), \omega \geq 0, \mathscr{H}_{1}$ be a positive $\mathfrak{q}, \omega$ integrable function defined on $[0, \infty)$, and $\mathcal{Q}$ and $\mathcal{U}$ be two $\mathfrak{q}, \omega$-differentiable functions defined on $[0, \infty)$ such that $\widehat{Q}^{\prime} \in L_{s}([0, \infty))$ and $\mathcal{U}^{\prime} \in L_{r}([0, \infty))$. Then, the inequalities

$$
\begin{aligned}
& 2 \mid\left(\left({ }_{0^{+}} \mathscr{J}_{\mathbf{q}, \omega}^{\alpha} \mathscr{H}_{1}\right)(\varsigma)\left({ }_{0^{+}} \mathscr{I}_{\mathbf{q}, \omega}^{\alpha} Q \mathcal{U}\right)(\varsigma)\right. \\
& \left.-\left({ }_{0^{+}} \mathscr{I}_{\mathbf{q}, \omega}^{\alpha} \mathscr{H}_{1} \mathcal{Q}\right)(\varsigma)\left({ }_{0^{+}} \mathscr{J}_{\mathbf{q}, \omega}^{\alpha} \mathscr{H}_{1} \mathscr{U}\right)(\varsigma)\right) \mid \\
& \leq\left(\frac{\left\|Q^{\prime}\right\|_{s}^{u}}{\Gamma_{\mathfrak{q}}^{u}(\alpha)} \int_{0}^{\varsigma} \int_{0}^{\varsigma}\left(\varsigma-{ }_{\sigma} \Psi_{\mathfrak{q}}(x)\right)_{\sigma}^{\alpha-1}\left(\varsigma-{ }_{\sigma} \Psi_{\mathfrak{q}}(y)\right)_{\sigma}^{(\alpha-1)} \Phi^{\prime}(x)\right. \\
& \text { - } \left.\Phi^{\prime}(y) \mathscr{H}_{1}(x) \mathscr{H}_{1}(y)|x-y|^{1 / s_{1}+1 / r_{1}} d_{\mathfrak{q}, \omega} x d_{\mathfrak{q}, \omega} y\right)^{1 / u} \\
& \times\left(\frac{\left\|\mathcal{U}^{\prime}\right\|_{r}^{u_{1}}}{\Gamma_{\mathfrak{q}}^{u_{1}}(\alpha)} \int_{0}^{\varsigma} \int_{0}^{\varsigma}\left(\varsigma-{ }_{\sigma} \Psi_{\mathfrak{q}}(x)\right)_{\sigma}^{\alpha-1}\left(\varsigma-{ }_{\sigma} \Psi_{\mathfrak{q}}(y)\right)_{\sigma}^{\alpha-1}\right. \\
& \left.\cdot \mathscr{H}_{1}(x) \mathscr{H}_{1}(y)|x-y|^{1 / s_{1}+1 / r_{1}} d_{\mathfrak{q}, \omega} x d_{\mathfrak{q}, \omega} y\right)^{1 / u_{1}} \\
& \leq \frac{\left\|Q^{\prime}\right\|_{s}^{u}\left\|\mathcal{U}^{\prime}\right\|_{r}^{u_{1}}}{\left(\Gamma_{\mathfrak{q}}(\alpha)\right)^{2}}\left(\int_{0}^{\varsigma} \int_{0}^{\varsigma}\left(\varsigma-{ }_{\sigma} \Psi_{\mathfrak{q}}(x)\right)_{\sigma}^{\alpha-1}\left(\varsigma-{ }_{\sigma} \Psi_{\mathfrak{q}}(y)\right)_{\sigma}^{\alpha-1}\right. \\
& \left.\cdot \mathscr{H}_{1}(x) \mathscr{H}_{1}(y)|x-y|^{1 / s_{1}+1 / r_{1}} d_{\mathfrak{q}, \omega} x d_{\mathfrak{q}, \omega} y\right)
\end{aligned}
$$

hold for all $\varsigma>0$.

Proof. Let $x, y \in(0, \varsigma)$ and

$$
\mathscr{G}(x, y)=(\mathscr{Q}(x)-\mathscr{Q}(y))(\mathcal{U}(x)-\mathscr{U}(y)) .
$$

Then, $\mathscr{G}(x, y)$ can be written as

$$
\mathscr{G}(x, y)=\mathscr{Q}(x) \mathcal{U}(x)-\mathscr{Q}(x) \mathcal{U}(y)-\mathscr{Q}(y) \mathcal{U}(x)-\mathcal{U}(y) \mathscr{Q}(y) .
$$


Multiplying both sides of (21) by $\left(\left(\varsigma-{ }_{\sigma} \Psi_{\mathfrak{q}}(x)\right)_{\sigma}^{\alpha-1} / \Gamma_{\mathfrak{q}}(\alpha)\right)$ $\mathscr{H}_{1}(x)$ and then performing the $\mathfrak{q}, \omega$-integration with respect to $x$ over $(0, \varsigma)$, we have

$$
\begin{aligned}
\int_{0}^{\varsigma} \frac{\left(\varsigma-{ }_{\sigma} \Psi_{\mathfrak{q}}(x)\right)_{\sigma}^{\alpha-1}}{\Gamma_{\mathfrak{q}}(\alpha)} \mathscr{H}_{1}(x) \mathscr{G}(x, y) d_{\mathfrak{q}, \omega} x \\
=\int_{0}^{\varsigma} \frac{\left(\varsigma-{ }_{\sigma} \Psi_{\mathfrak{q}}(x)\right)_{\sigma}^{\alpha-1}}{\Gamma_{\mathfrak{q}}(\alpha)} \mathscr{H}_{1}(x) \mathscr{Q}(x) \mathscr{U}(x) d_{\mathfrak{q}, \omega} x \\
\quad-\int_{0}^{\varsigma} \frac{\left(\varsigma-{ }_{\sigma} \Psi_{\mathfrak{q}}(x)\right)_{\sigma}^{\alpha-1}}{\Gamma_{\mathfrak{q}}(\alpha)} \mathscr{H}_{1}(x) \mathcal{Q}(x) \mathscr{U}(y) d_{\mathfrak{q}, \omega} x \\
\quad-\int_{0}^{\varsigma} \frac{\left(\varsigma-{ }_{\sigma} \Psi_{\mathfrak{q}}(x)\right)_{\sigma}^{\alpha-1}}{\Gamma_{\mathfrak{q}}(\alpha)} \mathscr{H}_{1}(x) \mathscr{Q}(y) \mathscr{U}(x) d_{\mathfrak{q}, \omega} x \\
\quad-\mathscr{U}(y) \mathscr{Q}(y) \int_{0}^{\varsigma} \frac{\left(\varsigma-{ }_{\sigma} \Psi_{\mathfrak{q}}(x)\right)_{\sigma}^{\alpha-1}}{\Gamma_{\mathfrak{q}}(\alpha)} \mathscr{H}_{1}(x) d_{\mathfrak{q}, \omega} x .
\end{aligned}
$$

Inequality (22) can be rewritten as

$$
\begin{aligned}
\int_{0}^{\varsigma} \frac{\left(\varsigma-{ }_{\sigma} \Psi_{\mathfrak{q}}(x)\right)_{\sigma}^{\alpha-1}}{\Gamma_{\mathfrak{q}}(\alpha)} \mathscr{H}_{1}(x) \mathscr{G}(x, y) d_{\mathfrak{q}, \omega} x \\
=\left(0_{0^{+}} \mathscr{J}_{\mathfrak{q}, \omega}^{\alpha} \mathscr{H}_{1} \mathscr{Q} \mathscr{U}\right)(\varsigma)-\mathscr{U}(y)\left({ }_{0^{+}} \mathscr{J}_{\mathfrak{q}, \omega}^{\alpha} \mathscr{H}_{1} \mathcal{Q}\right)(\varsigma) \\
\quad-\mathbb{Q}(y)\left({ }_{0^{+}} \mathscr{J}_{\mathfrak{q}, \omega}^{\alpha} \mathscr{H}_{1} \mathscr{U}\right)(\varsigma)+\mathscr{Q}(y) \mathscr{U}(y)\left({ }_{0^{+}} \mathscr{J}_{\mathfrak{q}, \omega}^{\alpha} \mathscr{H}_{1}\right)(\varsigma) .
\end{aligned}
$$

Multiplying both sides of (23) by $\left(\left(\varsigma-{ }_{\sigma} \Psi_{\mathfrak{q}}(y)\right)_{\sigma}^{\alpha-1} / \Gamma_{\mathfrak{q}}(\alpha)\right)$ $\mathscr{H}_{1}(y)$ and then performing the $\mathfrak{q}, \omega$-integration with respect to $y$ over $(0, \varsigma)$, we get

$$
\begin{gathered}
\frac{1}{\left(\Gamma_{\mathfrak{q}}(\alpha)\right)^{2}} \int_{0}^{\varsigma} \int_{0}^{\varsigma}\left(\varsigma-{ }_{\sigma} \Psi_{\mathfrak{q}}(x)\right)_{\sigma}^{\alpha-1}\left(\varsigma-{ }_{\sigma} \Psi_{\mathfrak{q}}(y)\right)_{\sigma}^{\alpha-1} \\
\quad \mathscr{H}_{1}(x) \mathscr{H}_{1}(y) \mathscr{G}(x, y) d_{\mathfrak{q}, \omega} x d_{\mathfrak{q}, \omega} y \\
=2\left(\left(0_{0^{+}} \mathscr{\mathscr { J }}_{\mathfrak{q}, \omega}^{\alpha} \mathscr{H}_{1}\right)(\varsigma)\left({ }_{0^{+}} \mathscr{\mathscr { J }}_{\mathfrak{q}, \omega}^{\alpha} Q \mathcal{U}\right)(\varsigma)\right. \\
\left.\quad-\left({ }_{0^{+}} \mathscr{J}_{\mathfrak{q}, \omega}^{\alpha} \mathscr{H}_{1} \mathcal{Q}\right)(\varsigma)\left({ }_{0^{+}} \mathscr{J}_{\mathfrak{q}, \omega}^{\alpha} \mathscr{H}_{1} \mathscr{U}\right)(\varsigma)\right) .
\end{gathered}
$$

Similarly, we have

$$
\mathscr{G}(x, y)=\int_{x}^{y} \int_{x}^{y} Q^{\prime}(\theta) \mathcal{U}^{\prime}(\vartheta) d \theta d \vartheta
$$

Taking into account the Hölder inequality, we have

$$
|Q(x)-\mathscr{Q}(y)| \leq\left.\left.|x-y|^{1 / s_{1}}\left|\int_{x}^{y}\right| Q^{\prime}(\theta)\right|^{s} d \theta\right|^{1 / s},
$$

$$
|\mathcal{U}(x)-\mathscr{U}(y)| \leq\left.\left.|x-y|^{1 / r_{1}}\left|\int_{x}^{y}\right| \mathcal{U}^{\prime}(\vartheta)\right|^{r} d \vartheta\right|^{1 / r} .
$$

It follows from (26) and (27) that

$$
\begin{aligned}
|\mathscr{G}(x, y)| & \leq|(\mathscr{Q}(x)-\mathscr{Q}(y))(\mathcal{U}(x)-\mathscr{U}(y))| \\
& \leq\left.\left.\left.\left.|x-y|^{1 / s_{1}+1 / r_{1}}\left|\int_{x}^{y}\right| \mathscr{Q}^{\prime}(\theta)\right|^{s} d \theta\right|^{1 / s}\left|\int_{x}^{y}\right| \mathcal{U}^{\prime}(\mathcal{\vartheta})\right|^{r} d \mathcal{V}\right|^{1 / r} .
\end{aligned}
$$

From (24) and (28), we obtain

$$
\begin{aligned}
2 \mid\left(\left({ }_{0^{+}}\right.\right. & \left.\mathscr{J}_{\mathfrak{q}, \omega}^{\alpha} \mathscr{H}_{1}\right)(\varsigma)\left({ }_{0^{+}} \mathscr{J}_{\mathfrak{q}, \omega}^{\alpha} \mathscr{Q} \mathscr{U}\right)(\varsigma) \\
& \left.-\left({ }_{0}^{+} \mathscr{J}_{\mathfrak{q}, \omega}^{\alpha} \mathscr{H}_{1} \mathscr{Q}\right)(\varsigma)\left({ }_{0^{+}} \mathscr{J}_{\mathfrak{q}, \omega}^{\alpha} \mathscr{H}_{1} \mathscr{U}\right)(\varsigma)\right) \mid \\
= & \frac{1}{\left(\Gamma_{\mathfrak{q}}(\alpha)\right)^{2}} \int_{0}^{\varsigma} \int_{0}^{\varsigma}\left(\varsigma-{ }_{\sigma} \Psi_{\mathfrak{q}}(x)\right)_{\sigma}^{\alpha-1}\left(\varsigma-{ }_{\sigma} \Psi_{\mathfrak{q}}(y)\right)_{\sigma}^{\alpha-1} \\
& \cdot \mathscr{H}_{1}(x) \mathscr{H}_{1}(y)|\mathscr{G}(x, y)| d_{\mathfrak{q}, \omega} x d_{\mathfrak{q}, \omega} y \\
\leq & \frac{1}{\left(\Gamma_{\mathfrak{q}}(\alpha)\right)^{2}} \int_{0}^{\varsigma} \int_{0}^{\varsigma}\left(\varsigma-{ }_{\sigma} \Psi_{\mathfrak{q}}(x)\right)_{\sigma}^{\alpha-1}\left(\varsigma-{ }_{\sigma} \Psi_{\mathfrak{q}}(y)\right)_{\sigma}^{\alpha-1} \mathscr{H}_{1}(x) \mathscr{H}_{1}(y) \\
& \times\left.\left.\left.\left.|x-y|^{1 / s_{1}+1 / r_{1}}\left|\int_{x}^{y}\right| \mathbb{Q}^{\prime}(\theta)\right|^{s} d \theta\right|^{1 / s}\left|\int_{x}^{y}\right| \mathscr{U}^{\prime}(\vartheta)\right|^{r} d \vartheta\right|^{1 / r} d_{\mathfrak{q}, \omega} x d_{\mathfrak{q}, \omega} y .
\end{aligned}
$$

Making use of the Hölder inequality for bivariate integral, we have

$$
\begin{aligned}
& 2 \mid\left(\left({ }_{0^{+}} \mathscr{J}_{\mathrm{q}, \omega}^{\alpha} \mathscr{H}_{1}\right)(\varsigma)\left({ }_{0^{+}} \mathscr{J}_{\mathbf{q}, \omega}^{\alpha} \mathcal{Q} \mathcal{U}\right)(\varsigma)\right. \\
& \left.-\left({ }_{0^{+}} \mathscr{J}_{\mathbf{q}, \omega}^{\alpha} \mathscr{H}_{1} \mathcal{Q}\right)(\varsigma)\left({ }_{0^{+}} \mathscr{I}_{\mathbf{q}, \omega}^{\alpha} \mathscr{H}_{1} \mathscr{U}\right)(\varsigma)\right) \mid \\
& \leq \frac{1}{\left(\Gamma_{\mathfrak{q}}(\alpha)\right)^{2}}\left(\int_{0}^{\varsigma} \int_{0}^{\varsigma}\left(\varsigma-{ }_{\sigma} \Psi_{\mathfrak{q}}(x)\right)_{\sigma}^{\alpha-1}\right. \\
& \cdot\left(\varsigma-{ }_{\sigma} \Psi_{\mathfrak{q}}(y)\right)_{\sigma}^{\alpha-1} \mathscr{H}_{1}(x) \mathscr{H}_{1}(y) \\
& \left.\times\left.\left.|x-y|^{1 / s_{1}+1 / r_{1}}\left|\int_{x}^{y}\right| Q^{\prime}(\theta)\right|^{s} d \theta\right|^{u / s} d_{\mathfrak{q}, \omega} x d_{\mathfrak{q}, \omega} y\right)^{1 / u} \\
& \times\left(\int_{0}^{\varsigma} \int_{0}^{\varsigma}\left(\varsigma-{ }_{\sigma} \Psi_{\mathfrak{q}}(x)\right)_{\sigma}^{\alpha-1}\left(\varsigma-{ }_{\sigma} \Psi_{\mathfrak{q}}(y)\right)_{\sigma}^{\alpha-1} \mathscr{H}_{1}(x) \mathscr{H}_{1}(y)\right. \\
& \left.\times\left.\left.|x-y|^{1 / s_{1}+1 / r_{1}}\left|\int_{x}^{y}\right| \mathcal{U}^{\prime}(\mathcal{\vartheta})\right|^{r} d \vartheta\right|^{u_{1} / r} d_{\mathfrak{q}, \omega} x d_{\mathfrak{q}, \omega} y\right)^{1 / u_{1}} .
\end{aligned}
$$

It follows from (30) and the inequalities

$$
\left.\left.\left|\int_{x}^{y}\right| \mathcal{Q}^{\prime}(\theta)\right|^{s} d \theta\right|^{1 / s} \leq\left\|Q^{\prime}\right\|_{s^{\prime}},\left.\left.\left|\int_{x}^{y}\right| \mathcal{U}^{\prime}(\vartheta)\right|^{r} d \vartheta\right|^{1 / r} \leq \mathrm{a}\left\|\mathcal{U}^{\prime}\right\|_{r}
$$


that

$$
\begin{aligned}
2 \mid\left(\left(0_{0^{+}}\right.\right. & \left.\mathscr{J}_{\mathfrak{q}, \omega}^{\alpha} \mathscr{H}_{1}\right)(\varsigma)\left({ }_{0^{+}} \mathscr{J}_{\mathfrak{q}, \omega}^{\alpha} Q \mathcal{U}\right)(\varsigma) \\
& \left.-\left({ }_{0^{+}} \mathscr{J}_{\mathfrak{q}, \omega}^{\alpha} \mathscr{H}_{1} Q\right)(\varsigma)\left({ }_{0^{+}} \mathcal{F}_{\mathfrak{q}, \omega}^{\alpha} \mathscr{H}_{1} \mathcal{U}\right)(\varsigma)\right) \mid \\
\leq & \left(\frac{\left\|\mathcal{Q}^{\prime}\right\|_{s}^{u}}{\Gamma_{\mathfrak{q}}^{u}(\alpha)} \int_{0}^{\varsigma} \int_{0}^{\varsigma}\left(\varsigma-{ }_{\sigma} \Psi_{\mathfrak{q}}(x)\right)_{\sigma}^{\alpha-1}\left(\varsigma_{-}{ }_{\sigma} \Psi_{\mathfrak{q}}(y)\right)_{\sigma}^{\alpha-1} \Phi^{\prime}(x) \Phi^{\prime}(y)\right. \\
& \left.\cdot \mathscr{H}_{1}(x) \mathscr{H}_{1}(y)|x-y|^{1 / s_{1}+1 / r_{1}} d_{\mathfrak{q}, \omega} x d_{\mathfrak{q}, \omega} y\right)^{1 / u} \\
& \times\left(\frac{\left\|\mathcal{U}^{\prime}\right\|_{r}^{u_{1}}}{\Gamma_{\mathfrak{q}}^{u_{1}}(\alpha)} \int_{0}^{\varsigma} \int_{0}^{\varsigma}\left(\varsigma-{ }_{\sigma} \Psi_{\mathfrak{q}}(x)\right)_{\sigma}^{\alpha-1}\left(\varsigma-{ }_{\sigma} \Psi_{\mathfrak{q}}(y)\right)_{\sigma}^{\alpha-1}\right. \\
& \left.\cdot \mathscr{H}_{1}(x) \mathscr{H}_{1}(y)|x-y|^{1 / s_{1}+1 / r_{1}} d_{\mathfrak{q}, \omega} x d_{\mathfrak{q}, \omega} y\right)^{1 / u_{1}} .
\end{aligned}
$$

Therefore, we get the desired inequality

$$
\begin{aligned}
2 \mid\left(\left({ }_{0^{+}}\right.\right. & \left.\mathscr{J}_{\mathfrak{q}, \omega}^{\alpha} \mathscr{H}_{1}\right)(\varsigma)\left({ }_{0^{+}} \mathscr{J}_{\mathfrak{q}, \omega}^{\alpha} \mathcal{Q} \mathcal{U}\right)(\varsigma) \\
& \left.-\left({ }_{0^{+}} \mathscr{F}_{\mathfrak{q}, \omega}^{\alpha} \mathscr{H}_{1} Q\right)(\varsigma)\left({ }_{0^{+}} \mathscr{J}_{\mathfrak{q}, \omega}^{\alpha} \mathscr{H}_{1} \mathscr{U}\right)(\varsigma)\right) \mid \\
\leq & \frac{\left\|\mathcal{Q}^{\prime}\right\|_{s}^{u}\left\|\mathscr{U}^{\prime}\right\|_{r}^{u_{1}}}{\left(\Gamma_{\mathfrak{q}_{1}}(\alpha)\right)^{2}}\left(\int_{0}^{\varsigma} \int_{0}^{\varsigma}\left(\varsigma-{ }_{\sigma} \Psi_{\mathfrak{q}}(x)\right)_{\sigma}^{(\alpha-1)}\left(\varsigma-{ }_{\sigma} \Psi_{\mathfrak{q}}(y)\right)_{\sigma}^{\alpha-1}\right. \\
& \left.\cdot \mathscr{H}_{1}(x) \mathscr{H}_{1}(y)|x-y|^{1 / s_{1}+1 / r_{1}} d_{\mathfrak{q}, \omega} x d_{\mathfrak{q}, \omega} y\right) .
\end{aligned}
$$

Let $\omega=1$. Then, Theorem 7 leads to Corollary 8 which provide a new result for $\mathfrak{q}$-fractional integral operator.hold for all $\varsigma>0$.

Corollary 8. Let $s, s_{1}, u, u_{1}, r, r_{1}>1$ with $s^{-1}+s_{1}^{-1}=r^{-1}+$ $r_{1}^{-1}=u^{-1}+u_{1}^{-1}=1, \quad \mathfrak{q} \in(0,1), \quad \mathscr{H}_{1}$ be a positive $\mathfrak{q}, 1$ integrable function defined on $[0, \infty)$, and $Q$ and $\mathcal{U}$ be two $\mathfrak{q}, 1$-differentiable functions defined on $[0, \infty)$ such that $Q^{\prime} \in L_{s}([0, \infty))$ and $\mathcal{U}^{\prime} \in L_{r}([0, \infty))$. Then, the inequalities

$$
\begin{aligned}
& 2 \mid\left(\left({ }_{0^{+}} \mathscr{J}_{\mathbf{q}, 1}^{\alpha} \mathscr{H}_{1}\right)(\varsigma)\left({ }_{0^{+}} \mathscr{J}_{\mathfrak{q}, 1}^{\alpha} \mathcal{Q} \mathcal{U}\right)(\varsigma)\right. \\
& \left.-\left({ }_{0^{+}} \mathscr{J}_{\mathfrak{q}, 1}^{\alpha} \mathscr{H}_{1} \mathcal{Q}\right)(\varsigma)\left({ }_{0^{+}} \mathscr{F}_{\mathfrak{q}, 1}^{\alpha} \mathscr{H}_{1} \mathscr{U}\right)(\varsigma)\right) \mid \\
& \leq\left(\frac{\left\|Q^{\prime}\right\|_{s}^{u}}{\Gamma_{\mathfrak{q}}^{u}(\alpha)} \int_{0}^{\varsigma} \int_{0}^{\varsigma}\left(\varsigma-{ }_{\sigma} \Psi_{\mathfrak{q}}(x)\right)_{\sigma}^{(\alpha-1)}\left(\varsigma-{ }_{\sigma} \Psi_{\mathfrak{q}}(y)\right)_{\sigma}^{\alpha-1}\right. \\
& \text {. } \left.\Phi^{\prime}(x) \Phi^{\prime}(y) \mathscr{H}_{1}(x) \mathscr{H}_{1}(y)|x-y|^{1 / s_{1}+1 / r_{1}} d_{q} x d_{\mathfrak{q}} y\right)^{1 / u} \\
& \times\left(\frac{\left\|\mathcal{U}^{\prime}\right\|_{r}^{u_{1}}}{\Gamma_{\mathfrak{q}}^{u_{1}}(\alpha)} \int_{0}^{\varsigma} \int_{0}^{\varsigma}\left(\varsigma-{ }_{\sigma} \Psi_{\mathfrak{q}}(x)\right)_{\sigma}^{\alpha-1}\left(\varsigma-{ }_{\sigma} \Psi_{\mathfrak{q}}(y)\right)_{\sigma}^{\alpha-1}\right. \\
& \left.\cdot \mathscr{H}_{1}(x) \mathscr{H}_{1}(y)|x-y|^{1 / s_{1}+1 / r_{1}} d_{\mathfrak{q}} x d_{\mathfrak{q}} y\right)^{1 / u_{1}} \\
& \leq \frac{\left\|Q^{\prime}\right\|_{s}^{u}\left\|\mathcal{U}^{\prime}\right\|_{r}^{u_{1}}}{\left(\Gamma_{\mathfrak{q}}(\alpha)\right)^{2}}\left(\int_{0}^{\varsigma} \int_{0}^{\varsigma}\left(\varsigma-{ }_{\sigma} \Psi_{\mathfrak{q}}(x)\right)_{\sigma}^{\alpha-1}\left(\varsigma-{ }_{\sigma} \Psi_{\mathfrak{q}}(y)\right)_{\sigma}^{\alpha-1}\right. \\
& \left.\cdot \mathscr{H}_{1}(x) \mathscr{H}_{1}(y)|x-y|^{1 / s_{1}+1 / r_{1}} d_{\mathfrak{q}} x d_{\mathfrak{q}} y\right)
\end{aligned}
$$

hold for all $\varsigma>0$.
Remark 9. If $\mathfrak{q}=\omega=1$, then Theorem 7 reduces to the result for the Riemann-Liouville fractional integral operator given in [77]. Some results given in the literature $[13,78]$ can also be obtained from Theorem 7 immediately.

Theorem 10. Let $s, s_{1}, u, u_{1}, r, r_{1}>1$ with $s^{-1}+s_{1}^{-1}=r^{-1}+$ $r_{1}^{-1}=u^{-1}+u_{1}^{-1}=1, \mathfrak{q}_{i} \in(0,1), \omega_{i} \geq 0(i=1,2), \mathscr{H}_{1}$ and $\mathscr{H}_{2}$ be the positive $\mathfrak{q}_{i}, \omega_{i}$-integrable functions defined on $[0, \infty)$, and $\mathcal{Q}$ and $\mathcal{U}$ be the $\mathfrak{q}_{i}, \omega_{i}$-differentiable functions defined on $[0, \infty)$ such that $Q^{\prime} \in L_{s}([0, \infty))$ and $\mathcal{U}^{\prime} \in L_{r}([0, \infty))$. Then, the inequality

$$
\begin{aligned}
& \mathrm{I}\left({ }_{0^{+}} \mathscr{J}_{\mathfrak{q}_{2}, \omega_{2}}^{\beta} \mathscr{H}_{2}\right)(\varsigma)\left(0_{0^{+}} \mathscr{J}_{\mathfrak{q}_{1}, \omega_{1}}^{\alpha} \mathscr{H}_{1} \mathcal{Q} \mathscr{U}\right)(\varsigma) \\
& -\left({ }_{0^{+}} \mathscr{J}_{\mathfrak{q}_{2}, \omega_{2}}^{\beta} \mathscr{H}_{1} \mathscr{U}\right)(\varsigma)\left({ }_{0^{+}} \mathscr{J}_{\mathbf{q}_{1}, \omega_{1}}^{\alpha} \mathscr{H}_{1} \mathcal{Q}\right)(\varsigma) \\
& -\left({ }_{0^{+}} \mathscr{J}_{\mathfrak{q}_{2}, \omega_{2}}^{\beta} \mathscr{H}_{2} \mathcal{Q}\right)(\varsigma)\left({ }_{0^{+}} \mathscr{F}_{\mathfrak{q}_{1}, \omega_{1}}^{\alpha} \mathscr{H}_{1} \mathscr{U}\right)(\varsigma) \\
& +\left({ }_{0^{+}} \mathscr{J}_{\mathfrak{q}_{2}, \omega_{2}}^{\beta} \mathscr{H}_{2} \mathcal{Q} \mathscr{U}\right)(\varsigma)\left(0_{0^{+}} \mathscr{J}_{\mathfrak{q}_{1}, \omega_{1}}^{\alpha} \mathscr{H}_{1}\right)(\varsigma) \mid \\
& \leq \frac{\|Q\|_{s}\|\mathcal{U}\|_{r}}{\Gamma_{\mathfrak{q}_{1}}(\alpha) \Gamma_{\mathfrak{q}_{2}}(\beta)} \int_{0}^{\varsigma} \int_{0}^{\varsigma}\left(\varsigma-\sigma_{\sigma_{1}} \Psi_{\mathfrak{q}_{1}}(x)\right)_{\sigma_{1}}^{\alpha-1}\left(\varsigma-\sigma_{\sigma_{2}} \Psi_{\mathfrak{q}_{2}}(y)\right)_{\sigma_{2}}^{\beta-1} \\
& \times|x-y|^{1 / s_{1}+1 / r_{1}} \mathscr{H}_{1}(x) \mathscr{H}_{2}(y) d_{\mathfrak{q}_{1}, \omega_{1}} x d_{\mathfrak{q}_{2}, \omega_{2}} y
\end{aligned}
$$

holds for all $\varsigma>0$.

Proof. Multiplying both sides of (23) by $\left(\left(\varsigma-{ }_{\sigma_{2}} \Psi_{\mathfrak{q}_{2}}(y)\right)_{\sigma_{2}}^{\beta-1} /\right.$ $\left.\Gamma_{\mathfrak{q}_{2}(\beta)}\right) \mathscr{H}_{2}(y)$ and performing $\mathfrak{q}_{2}, \omega_{2}$-integration with respect to $y$ over $(0, \varsigma)$, we have

$$
\begin{aligned}
& \frac{1}{\Gamma_{\mathfrak{q}_{1}}(\alpha) \Gamma_{\mathfrak{q}_{2}}(\beta)} \int_{0}^{\varsigma} \int_{0}^{\varsigma}\left(\varsigma-{ }_{\sigma_{1}} \Psi_{\mathfrak{q}_{1}}(x)\right)_{\sigma_{1}}^{\alpha-1}\left(\varsigma_{-\sigma_{2}} \Psi_{\mathfrak{q}_{2}}(y)\right)_{\sigma_{2}}^{\beta-1} \\
& \text { - } \mathscr{H}_{1}(x) \mathscr{H}_{2}(y) \mathscr{G}(x, y) d_{\mathfrak{q}_{1}, \omega_{1}} x d_{\mathfrak{q}_{2}, \omega_{29}} y \\
& =\left({ }_{0^{+}} \mathscr{J}_{\mathbf{q}_{2}, \omega_{2}}^{\beta} \mathscr{H}_{2}\right)(\varsigma)\left({ }_{0^{+}} \mathscr{\mathscr { I }}_{\mathbf{q}_{1}, \omega_{1}}^{\alpha} \mathscr{H}_{1} \mathscr{Q} \mathscr{U}\right)(\varsigma) \\
& -\left({ }_{0^{+}} \mathscr{J}_{\mathfrak{q}_{2}, \omega_{2}}^{\beta} \mathscr{H}_{1} \mathcal{U}\right)(\varsigma)\left(0_{0^{+}} \mathscr{J}_{\mathfrak{q}_{1}, \omega_{1}}^{\alpha} \mathscr{H}_{1} \mathcal{Q}\right)(\varsigma) \\
& -\left({ }_{0^{+}} \mathscr{J}_{\mathfrak{q}_{2}, \omega_{2}}^{\beta} \mathscr{H}_{2} \mathcal{Q}\right)(\varsigma)\left({ }_{0^{+}} \mathscr{J}_{\mathfrak{q}_{1}, \omega_{1}}^{\alpha} \mathscr{H}_{1} \mathscr{U}\right)(\varsigma) \\
& +\left({ }_{0^{+}} \mathscr{J}_{\mathbf{q}_{2}, \omega_{2}}^{\beta} \mathscr{H}_{2} \mathscr{Q} \mathscr{U}\right)(\varsigma)\left(0_{0^{+}} \mathscr{J}_{\mathbf{q}_{1}, \omega_{1}}^{\alpha} \mathscr{H}_{1}\right)(\varsigma) .
\end{aligned}
$$

Taking modulus on both sides of (36), one has

$$
\begin{aligned}
& \mathrm{I}\left(0_{0^{+}} \mathscr{J}_{\mathfrak{q}_{2}, \omega_{2}}^{\beta} \mathscr{H}_{2}\right)(\varsigma)\left(0_{0^{+}} \mathscr{J}_{\mathbf{q}_{1}, \omega_{1}}^{\alpha} \mathscr{H}_{1} \mathcal{Q} \mathscr{U}\right)(\varsigma) \\
& -\left({ }_{0^{+}} \mathscr{J}_{\mathfrak{q}_{2}, \omega_{2}}^{\beta} \mathscr{H}_{1} \mathscr{U}\right)(\varsigma)\left({ }_{0^{+}} \mathscr{J}_{\mathbf{q}_{1}, \omega_{1}}^{\alpha} \mathscr{H}_{1} \mathcal{Q}\right)(\varsigma) \\
& -\left({ }_{0^{+}} \mathscr{J}_{\mathfrak{q}_{2}, \omega_{2}}^{\beta} \mathscr{H}_{2} \mathcal{Q}\right)(\varsigma)\left(0_{0^{+}} \mathscr{J}_{\mathfrak{q}_{1}, \omega_{1}}^{\alpha} \mathscr{H}_{1} \mathscr{U}\right)(\varsigma) \\
& +\left(0_{0^{+}} \mathscr{J}_{\mathfrak{q}_{2}, \omega_{2}}^{\beta} \mathscr{H}_{2} Q \mathcal{U}\right)(\varsigma)\left(0_{0^{+}} \mathscr{J}_{\mathfrak{q}_{1}, \omega_{1}}^{\alpha} \mathscr{H}_{1}\right)(\varsigma) \mid \\
& =\frac{1}{\Gamma_{\mathfrak{q}_{1}}(\alpha) \Gamma_{\mathfrak{q}_{2}}(\beta)} \int_{0}^{\varsigma} \int_{0}^{\varsigma}\left(\varsigma-\sigma_{\sigma_{1}} \Psi_{\mathfrak{q}_{1}}(x)\right)_{\sigma_{1}}^{\alpha-1}\left(\varsigma-{ }_{\sigma_{2}} \Psi_{\mathfrak{q}_{2}}(y)\right)_{\sigma_{2}}^{\beta-1} \\
& \text { - } \mathscr{H}_{1}(x) \mathscr{H}_{2}(y)|\mathscr{G}(x, y)| d_{\mathfrak{q}_{1}, \omega_{1}} x d_{\mathfrak{q}_{2}, \omega_{2}} y \\
& \leq \frac{1}{\Gamma_{\mathfrak{q}_{1}}(\alpha) \Gamma_{\mathfrak{q}_{2}}(\beta)} \int_{0}^{\varsigma} \int_{0}^{\varsigma}\left(\varsigma-{ }_{\sigma_{1}} \Psi_{\mathfrak{q}_{1}}(x)\right)_{\sigma_{1}}^{\alpha-1}\left(\varsigma-{ }_{\sigma_{2}} \Psi_{\mathfrak{q}_{2}}(y)\right)_{\sigma_{2}}^{\beta-1} \\
& \times\left.\left.\left.\left.|x-y|^{1 / s_{1}+1 / r_{1}}\left|\int_{x}^{y}\right| \mathcal{Q}^{\prime}(\theta)\right|^{s} d \theta\right|^{1 / s}\left|\int_{x}^{y}\right| \mathcal{U}^{\prime}(\vartheta)\right|^{r} d \vartheta\right|^{1 / r} \\
& \text { - } \mathscr{H}_{1}(x) \mathscr{H}_{2}(y) d_{\mathfrak{q}_{1}, \omega_{1}} x d_{\mathfrak{q}_{2}, \omega_{2}} y \\
& =\frac{\|Q\|_{s}\|\mathcal{U}\|_{r}}{\Gamma_{\mathfrak{q}_{1}}(\alpha) \Gamma_{\mathbf{q}_{2}}(\beta)} \int_{0}^{\varsigma} \int_{0}^{\varsigma}\left(\varsigma-{ }_{\sigma_{1}} \Psi_{\mathfrak{q}_{1}}(x)\right)_{\sigma_{1}}^{\alpha-1}\left(\varsigma-{ }_{\sigma_{2}} \Psi_{\mathbf{q}_{2}}(y)\right)_{\sigma_{2}}^{\beta-1} \\
& \times|x-y|^{1 / s_{1}+1 / r_{1}} \mathscr{H}_{1}(x) \mathscr{H}_{2}(y) d_{\mathfrak{q}_{1}, \omega_{1}} x d_{\mathfrak{q}_{2}, \omega_{2}} y \text {. }
\end{aligned}
$$


Let $\omega_{i}=1$ for $i=1,2$. Then, Theorem 10 leads to Corollary 11 which provide a new result for $q_{i}$-fractional integral operator.

Corollary 11. Let $s, s_{1}, u, u_{1}, r, r_{1}>1$ with $s^{-1}+s_{1}{ }^{-1}=r^{-1}+$ $r_{1}^{-1}=u^{-1}+u_{1}^{-1}=1, \mathfrak{q}_{i} \in(0,1)(i=1,2), \mathscr{H}_{1}$ and $\mathscr{H}_{2}$ be the positive $\mathfrak{q}_{i}$-integrable functions defined on $[0, \infty)$, and $\mathbb{Q}$ and $\mathcal{U}$ be the $\mathfrak{q}_{i}$-differentiable functions defined on $[0, \infty)$ such that $Q^{\prime} \in L_{s}([0, \infty))$ and $\mathcal{U}^{\prime} \in L_{r}([0, \infty))$. Then, the inequality

$$
\begin{aligned}
& I\left(0_{0^{+}} \mathscr{J}_{\mathfrak{q}_{2}}^{\beta} \mathscr{H}_{2}\right)(\varsigma)\left({ }_{0^{+}} \mathscr{J}_{\mathfrak{q}_{1}}^{\alpha} \mathscr{H}_{1} Q \mathcal{Q}\right)(\varsigma) \\
& -\left({ }_{0^{+}} \mathscr{J}_{\mathfrak{q}_{2}}^{\beta} \mathscr{H}_{1} \mathscr{U}\right)(\varsigma)\left({ }_{0^{+}} \mathscr{I}_{\mathfrak{q}_{1}}^{\alpha} \mathscr{H}_{1} \mathcal{Q}\right)(\varsigma) \\
& -\left({ }_{0^{+}} \mathscr{J}_{\mathfrak{q}_{2}}^{\beta} \mathscr{H}_{2} \mathcal{Q}\right)(\varsigma)\left(0^{+} \mathscr{J}_{\mathfrak{q}_{1}}^{\alpha} \mathscr{H}_{1} \mathscr{U}\right)(\varsigma) \\
& +\left(0_{0^{+}} \mathscr{J}_{\mathfrak{q}_{2}}^{\beta} \mathscr{H}_{2} Q \mathcal{Q} \mathcal{U}\right)(\varsigma)\left(0_{0^{+}} \mathscr{J}_{\mathfrak{q}_{1}}^{\alpha} \mathscr{H}_{1}\right)(\varsigma) \mid \\
& \leq \frac{\|Q \mathcal{Q}\|_{s}\|\mathcal{U}\|_{r}}{\Gamma_{\mathfrak{q}_{1}}(\alpha) \Gamma_{\mathfrak{q}_{2}}(\beta)} \int_{0}^{\varsigma} \int_{0}^{\varsigma}\left(\varsigma-{ }_{\sigma_{1}} \Psi_{\mathfrak{q}_{1}}(x)\right)_{\sigma_{1}}^{\alpha-1}\left(\varsigma-{ }_{\sigma_{2}} \Psi_{\mathfrak{q}_{2}}(y)\right)_{\sigma_{2}}^{\beta-1} \\
& \times|x-y|^{1 / s_{1}+1 / r_{1}} \mathscr{H}_{1}(x) \mathscr{H}_{2}(y) d_{\mathfrak{q}_{1}} x d_{\mathfrak{q}_{2}} y
\end{aligned}
$$

holds for all $\varsigma>0$.

Remark 12. Let $\mathfrak{q}_{i}=\omega_{i}=1$. Then, Theorem 10 becomes Theorem 14 of [77].

\section{Some New Generalizations by Fractional Quantum Hahn Integral Operator}

Theorem 13. Let $r, s>1$ with $1 / r+1 / s=1, \mathfrak{q}_{i} \in(0,1)$ and $\omega_{i} \geq 0$ for $i=1,2$, and $\mathbb{Q}$ and $\mathcal{U}$ be the the positive $\mathfrak{q}_{i}$, $\omega_{i}$-integral functions. Then the following inequalities

$(A 1)(1 / r)\left({ }_{0^{+}} \mathcal{I}_{\mathfrak{q}_{1}, \omega_{1}}^{\alpha} Q^{r}\right)(\varsigma)\left({ }_{0^{+}} \mathcal{J}_{\mathfrak{q}_{2}, \omega_{2}}^{\beta} \mathscr{U}^{r}\right)(\varsigma)+(1 / s)\left({ }_{0^{+}} \mathscr{J}_{\mathfrak{q}_{1}, \omega_{1}}^{\alpha} \mathcal{U}^{s}\right)$ $(\varsigma)\left({ }_{0^{+}} \mathscr{J}_{\mathfrak{q}_{2}, \omega_{2}}^{\beta} Q^{s}\right)(\varsigma) \geq\left({ }_{0^{+}} \mathscr{J}_{\mathfrak{q}_{1}, \omega_{1}}^{\alpha} Q \mathcal{Q}\right)(\varsigma)\left({ }_{0^{+}} \mathscr{J}_{\mathfrak{q}_{2}, \omega_{2}}^{\beta} Q \mathcal{Q} \mathcal{U}\right)(\varsigma)$

$(A 2)(1 / r)\left({ }_{0^{+}} \mathscr{J}_{\mathbf{q}_{1}, \omega_{1}}^{\alpha} Q^{r}\right)(\varsigma)\left({ }_{0^{+}} \mathscr{J}_{\mathbf{q}_{2}, \omega_{2}}^{\beta} \mathcal{U}^{s}\right)(\varsigma)+(1 / s)\left({ }_{0^{+}} \mathscr{J}_{\mathfrak{q}_{1}, \omega_{1}}^{\alpha} \mathcal{U}^{s}\right)$ $(\varsigma)\left({ }_{0^{+}} \mathscr{J}_{\mathfrak{q}_{2}, \omega_{2}}^{\beta} Q^{r}\right)(\varsigma) \geq\left({ }_{0^{+}} \mathscr{J}_{\mathfrak{q}_{1}, \omega_{1}}^{\alpha} Q \mathcal{U}\right)(\varsigma)\left({ }_{0^{+}} \mathscr{J}_{\mathfrak{q}_{2}, \omega_{2}}^{\beta} Q^{r-1} \mathscr{U}^{s-1}\right)(\varsigma)$

$(A 3)(1 / r)\left({ }_{0^{+}} \mathcal{J}_{\mathbf{q}_{1}, \omega_{1}}^{\alpha} Q^{r}\right)(\varsigma)\left({ }_{0^{+}} \mathscr{J}_{\mathbf{q}_{2}, \omega_{2}}^{\beta} \mathscr{U}^{2}\right)(\varsigma)+(1 / s)\left({ }_{0^{+}} \mathscr{J}_{\mathfrak{q}_{1}, \omega_{1}}^{\alpha} Q^{2}\right)$ $(\varsigma)\left({ }_{0^{+}} \mathscr{J}_{\mathfrak{q}_{2}, \omega_{2}}^{\beta} \mathscr{U}^{s}\right)(\varsigma) \geq\left({ }_{0^{+}} \mathscr{J}_{\mathfrak{q}_{1}, \omega_{1}}^{\alpha} Q \mathcal{Q}\right)(\varsigma)\left({ }_{0^{+}} \mathscr{J}_{\mathbf{q}_{2}, \omega_{2}}^{\beta} Q^{2 / s} \mathscr{U}^{2 / r}\right)(\varsigma)$

$(A 4)(1 / r)\left({ }_{0^{+}} \mathscr{J}_{\mathbf{q}_{1}, \omega_{1}}^{\alpha} Q^{2}\right)(\varsigma)\left({ }_{0^{+}} \mathscr{J}_{\mathbf{q}_{2}, \omega_{2}}^{\beta} Q^{r}\right)(\varsigma)+(1 / s)\left({ }_{0^{+}} \mathscr{J}_{\mathbf{q}_{1}, \omega_{1}}^{\alpha} \mathscr{U}^{2}\right)$ $(\varsigma)\left({ }_{0^{+}} \mathscr{J}_{\mathfrak{q}_{2}, \omega_{2}}^{\beta} \mathcal{U}^{s}\right)(\varsigma) \geq\left({ }_{0^{+}} \mathscr{I}_{\mathfrak{q}_{1}, \omega_{1}}^{\alpha} Q^{2 / r} \mathcal{U}^{2 / s}\right)(\varsigma)\left({ }_{0^{+}} \mathscr{I}_{\mathfrak{q}_{2}, \omega_{2}}^{\beta} Q \mathcal{Q} \mathcal{U}\right)(\varsigma)$ hold for all $\varsigma>0$.

Proof. Considering the well-known Young inequality

$$
\frac{1}{r} \rho_{1}^{r}+\frac{1}{s} \rho_{2}^{s} \geq \rho_{1} \rho_{2}
$$

for all $\rho_{1}, \rho_{2} \geq 0$ and $r, s>1$ with $r^{-1}+s^{-1}=1$.

Substituting $\rho_{1}=\mathbb{Q}(x) \mathcal{U}(y)$ and $\rho_{2}=\mathscr{Q}(y) \mathcal{U}(x)(x, y>0)$ into (39) gives

$$
\frac{1}{r} Q^{r}(x) \mathcal{U}^{r}(y)+\frac{1}{s} \widehat{Q}^{s}(y) \mathcal{U}^{s}(x) \geq \mathscr{Q}(x) \mathcal{U}(y) \mathscr{Q}(y) \mathcal{U}(x) .
$$

Multiplying both sides of (40) by

$$
\frac{\left(\varsigma-{ }_{\sigma_{1}} \Psi_{\mathfrak{q}_{1}}(x)\right)_{\sigma_{1}}^{\alpha-1}\left(\varsigma-{ }_{\sigma_{2}} \Psi_{\mathfrak{q}_{2}}(y)\right)_{\sigma_{2}}^{\beta-1}}{\Gamma_{\mathfrak{q}_{1}}(\alpha) \Gamma_{\mathfrak{q}_{2}}(\beta)}
$$

and then performing the $\mathfrak{q}_{i}, \omega_{i}$-integration with respect to $x$ and $y$ over $(0, \varsigma)$ lead to the conclusion that

$$
\begin{aligned}
& \frac{1}{r} \int_{0}^{\varsigma} \frac{\left(\varsigma-{ }_{\sigma_{1}} \Psi_{\mathfrak{q}_{1}}(x)\right)_{\sigma_{1}}^{\alpha-1}\left(\varsigma-{ }_{\sigma_{2}} \Psi_{\mathfrak{q}_{2}}(y)\right)_{\sigma_{2}}^{\beta-1}}{\Gamma_{\mathfrak{q}_{1}}(\alpha) \Gamma_{\mathfrak{q}_{2}}(\beta)} \\
& \cdot Q^{r}(x) \mathcal{U}^{r}(y) d_{\mathfrak{q}_{1}, \omega_{1}} x d_{\mathfrak{q}_{2}, \omega_{2}} y \\
& +\frac{1}{s} \int_{0}^{\varsigma} \frac{\left(\varsigma-\sigma_{\sigma_{1}} \Psi_{\mathfrak{q}_{1}}(x)\right)_{\sigma_{1}}^{\alpha-1}\left(\varsigma-\sigma_{\sigma_{2}} \Psi_{\mathfrak{q}_{2}}(y)\right)_{\sigma_{2}}^{\beta-1}}{\Gamma_{\mathfrak{q}_{1}}(\alpha) \Gamma_{\mathfrak{q}_{2}}(\beta)} \\
& \text { - } Q^{s}(y) \mathcal{U}^{s}(x) d_{\mathfrak{q}_{1}, \omega_{1}} x d_{\mathfrak{q}_{2}, \omega_{2}} y \\
& \geq \int_{0}^{\varsigma} \frac{\left(\varsigma-\sigma_{1} \Psi_{\mathfrak{q}_{1}}(x)\right)_{\sigma_{1}}^{\alpha-1}\left(\varsigma-{ }_{\sigma_{2}} \Psi_{\mathfrak{q}_{2}}(y)\right)_{\sigma_{2}}^{\beta-1}}{\Gamma_{\mathfrak{q}_{1}}(\alpha) \Gamma_{\mathfrak{q}_{2}}(\beta)} \\
& \text { - } Q(x) \mathcal{U}(y) \mathcal{Q}(y) \mathcal{U}(x) d_{\mathfrak{q}_{1}, \omega_{1}} x d_{\mathfrak{q}_{2}, \omega_{2}} y \text {. }
\end{aligned}
$$

Consequently, we have

$$
\begin{aligned}
& \frac{1}{r}\left({ }_{0^{+}} \mathscr{J}_{\mathfrak{q}_{1}, \omega_{1}}^{\alpha} Q^{r}\right)(\varsigma)\left({ }_{0^{+}} \mathscr{I}_{\mathfrak{q}_{2}, \omega_{2}}^{\beta} \mathscr{U}^{r}\right)(\varsigma) \\
& +\frac{1}{s}\left(0^{+} \mathscr{J}_{\mathfrak{q}_{1}, \omega_{1}}^{\alpha} \mathcal{U}^{s}\right)(\varsigma)\left({ }_{0^{+}} \mathscr{I}_{\mathfrak{q}_{2}, \omega_{2}}^{\beta} Q^{s}\right)(\varsigma) \\
& \geq\left(0^{+} \mathscr{J}_{\mathfrak{q}_{1}, \omega_{1}}^{\alpha} \mathcal{Q} \mathcal{U}\right)(\varsigma)\left(0^{+} \mathscr{J}_{\mathfrak{q}_{2}, \omega_{2}}^{\beta} \mathcal{Q} \mathcal{U}\right)(\varsigma) \text {, }
\end{aligned}
$$

which implies $\left(A_{1}\right)$. The remaining inequalities can be derived by adopting the similar argument and accompanying the selection of parameters in Young inequality as follows:

$$
\begin{aligned}
& \left(A_{2}\right) \rho_{1}=\mathscr{Q}(x) / \mathscr{Q}(y), \rho_{2}=\mathscr{U}(x) / \mathscr{U}(y), \\
& \left(A_{3}\right) \rho_{1}=\mathbb{Q}(x) \mathcal{U}^{2 / r}(y), \rho_{2}=\mathbb{Q}^{2 / s}(x) \mathcal{U}(y), \\
& \left(A_{4}\right) \rho_{1}=\mathbb{Q}^{2 / r}(x) \mathcal{Q}(y), \rho_{2}=\mathcal{U}^{2 / s}(x) \mathcal{U}(y) .
\end{aligned}
$$

Theorem 14. Let $r, s>0$ with $r+s=1, \mathfrak{q}_{i} \in(0,1)$ and $\omega_{i} \geq 0$ for $i=1,2$, and $\mathcal{Q}$ and $\mathcal{U}$ be the positive $\mathfrak{q}_{i}, \omega_{i}$-integral functions defined on $[0, \infty)$. Then, the inequalities.

$$
\begin{aligned}
& \left(A_{5}\right) r\left(0_{0^{+}} \mathscr{J}_{\mathfrak{q}_{1}, \omega_{1}}^{\alpha} \mathcal{Q}\right)(\varsigma)\left(o_{0^{+}} \mathscr{J}_{\mathbf{q}_{2}, \omega_{2}}^{\beta} \mathcal{U}\right)(\varsigma)+s\left(o_{0^{+}} \mathscr{J}_{\mathbf{q}_{1}, \omega_{1}}^{\alpha} \mathcal{U}\right)(\varsigma)\left(o_{0^{+}} \mathscr{J}_{\mathbf{q}_{2}, \omega_{2}}^{\beta}\right. \\
& \mathbb{Q})(\varsigma) \geq\left({ }_{0^{+}} \mathscr{J}_{\mathfrak{q}_{1}, \omega_{1}}^{\alpha} Q^{r} \mathscr{U}^{s}\right)(\varsigma)\left({ }_{0^{+}} \mathscr{J}_{\mathfrak{q}_{2}, \omega_{2}}^{\beta} Q^{s} \mathscr{U}^{r}\right)(\varsigma) \\
& \left(A_{6}\right) r\left({ }_{0^{+}} \mathscr{J}_{\mathfrak{q}_{1}, \omega_{1}}^{\alpha} \mathcal{Q}\right)(\varsigma)\left({ }_{0^{+}} \mathscr{J}_{\mathbf{q}_{2}, \omega_{2}}^{\beta} \mathcal{U}\right)(\varsigma)+s\left({ }_{0^{+}} \mathscr{J}_{\mathbf{q}_{1}, \omega_{1}}^{\alpha} \mathcal{U}\right)(\varsigma)\left({ }_{0^{+}} \mathcal{J}_{\mathbf{q}_{2}, \omega_{2}}^{\beta}\right. \\
& \mathcal{Q})(\varsigma) \geq\left({ }_{0^{+}} \mathscr{J}_{\mathbf{q}_{1}, \omega_{1}}^{\alpha} Q^{r} \mathcal{U}^{s}\right)(\varsigma)\left({ }_{0^{+}} \mathscr{J}_{\mathfrak{q}_{2}, \omega_{2}}^{\beta} Q^{r-1} \mathcal{U}^{s-1}\right)(\varsigma) \\
& \left(A_{7}\right) r\left({ }_{0^{+}} \mathscr{I}_{\mathfrak{q}_{1}, \omega_{1}}^{\alpha} \mathcal{Q}\right)(\varsigma)\left({ }_{0^{+}} \mathscr{I}_{\mathfrak{q}_{2}, \omega_{2}}^{\beta} \mathscr{U}^{2 / r}\right)(\varsigma)+s\left({ }_{0^{+}} \mathscr{I}_{\mathfrak{q}_{1}, \omega_{1}}^{\alpha} \mathcal{U}\right)(\varsigma) \\
& \left({ }_{0^{+}} \mathscr{J}_{\mathfrak{q}_{2}, \omega_{2}}^{\beta} Q^{2 / s}\right)(\varsigma) \geq\left({ }_{0^{+}} \mathscr{J}_{\mathbf{q}_{1}, \omega_{1}}^{\alpha} Q^{r} \mathscr{U}^{s}\right)(\varsigma)\left({ }_{0^{+}} \mathscr{I}_{\mathfrak{q}_{2}, \omega_{2}}^{\beta} Q^{2} \mathscr{U}^{2}\right)(\varsigma) \\
& \left(A_{8}\right) r\left({ }_{0^{+}} \mathscr{J}_{\mathfrak{q}_{1}, \omega_{1}}^{\alpha} Q^{2 / r}\right)(\varsigma)\left({ }_{0^{+}} \mathscr{J}_{\mathfrak{q}_{2}, \omega_{2}}^{\beta} \mathcal{Q}\right)(\varsigma)+s\left({ }_{0^{+}} \mathscr{I}_{\mathfrak{q}_{1}, \omega_{1}}^{\alpha} \mathcal{U}^{2 / s}\right)(\varsigma) \\
& \left({ }_{0^{+}} \mathscr{J}_{\mathfrak{q}_{2}, \omega_{2}}^{\beta} \mathcal{U}\right)(\varsigma) \geq\left({ }_{0^{+}} \mathscr{J}_{\mathfrak{q}_{1}, \omega_{1}}^{\alpha} Q^{2} \mathcal{U}^{2}\right)(\varsigma)\left({ }_{0^{+}} \mathscr{J}_{\mathfrak{q}_{2}, \omega_{2}}^{\beta} Q^{s} \mathcal{U}^{r}\right)(\varsigma) \\
& \text { hold for all } \varsigma>0 \text {. }
\end{aligned}
$$

Proof. Considering the well-known weighted $A M-G M$ inequality

$$
r \rho_{1}+s \rho_{2} \geq \rho_{1}^{r} \rho_{2}^{s} \quad\left(\rho_{1}, \rho_{2} \geq 0, r, s>0, r+s=1\right) .
$$



yields

Substituting $\rho_{1}=\mathscr{Q}(x) \mathscr{U}(y)$ and $\rho_{2}=\mathscr{Q}(y) \mathscr{U}(x)(x, y>0)$

$$
r \mathscr{Q}(x) \mathscr{U}(y)+s \mathscr{Q}(y) \mathscr{U}(x) \geq \mathbb{Q}^{r}(x) \mathcal{U}^{r}(y) \mathscr{Q}^{s}(y) \mathcal{U}^{s}(x) .
$$

Conducting product on both sides of (45) by

$$
\frac{\left(\varsigma-\sigma_{1} \Psi_{\mathfrak{q}_{1}}(x)\right)_{\sigma_{1}}^{\alpha-1}\left(\varsigma-\sigma_{\sigma_{2}} \Psi_{\mathfrak{q}_{2}}(y)\right)_{\sigma_{2}}^{\beta-1}}{\Gamma_{\mathbf{q}_{1}}(\alpha) \Gamma_{\mathfrak{q}_{2}}(\beta)},
$$

and then performing the $\mathfrak{q}_{i}, \omega_{i}$-integration with respect to $x$ and $y$ over $(0, \varsigma)$, we obtain

$$
\begin{aligned}
& r \int_{0}^{\varsigma} \frac{\left(\varsigma-\sigma_{1} \Psi_{\mathfrak{q}_{1}}(x)\right)_{\sigma_{1}}^{\alpha-1}\left(\varsigma-\sigma_{\sigma_{2}} \Psi_{\mathfrak{q}_{2}}(y)\right)_{\sigma_{2}}^{\beta-1}}{\Gamma_{\mathfrak{q}_{1}}(\alpha) \Gamma_{\mathfrak{q}_{2}}(\beta)} \\
& \text { - } Q(x) \mathcal{U}(y) d_{\mathfrak{q}_{1}, \omega_{1}} x d_{\mathfrak{q}_{2}, \omega_{2}} y \\
& +s \int_{0}^{\varsigma} \frac{\left(\varsigma-\sigma_{1} \Psi_{\mathfrak{q}_{1}}(x)\right)_{\sigma_{1}}^{\alpha-1}\left(\varsigma-\sigma_{\sigma_{2}} \Psi_{\mathfrak{q}_{2}}(y)\right)_{\sigma_{2}}^{\beta-1}}{\Gamma_{\mathfrak{q}_{1}}(\alpha) \Gamma_{\mathbf{q}_{2}}(\beta)} \\
& \text { - Q }(y) \mathcal{U}(x) d_{\mathfrak{q}_{1}, \omega_{1}} x d_{\mathfrak{q}_{2}, \omega_{2}} y \\
& \geq \int_{0}^{\varsigma} \frac{\left(\varsigma-\sigma_{\sigma_{1}} \Psi_{\mathfrak{q}_{1}}(x)\right)_{\sigma_{1}}^{\alpha-1}\left(\varsigma-\sigma_{\sigma_{2}} \Psi_{\mathfrak{q}_{2}}(y)\right)_{\sigma_{2}}^{\beta-1}}{\Gamma_{\mathfrak{q}_{1}}(\alpha) \Gamma_{\mathfrak{q}_{2}}(\beta)} \\
& \text { - } \mathbb{Q}^{r}(x) \mathcal{U}^{r}(y) \mathcal{Q}^{s}(y) \mathcal{U}^{s}(x) d_{\mathfrak{q}_{1}, \omega_{1}} x d_{\mathfrak{q}_{2}, \omega_{2}} y \text {. }
\end{aligned}
$$

Consequently, we have

$$
\begin{aligned}
& r\left({ }_{0^{+}} \mathscr{J}_{\mathbf{q}_{1}, \omega_{1}}^{\alpha} \mathcal{Q}\right)(\varsigma)\left({ }_{0^{+}} \mathscr{J}_{\mathbf{q}_{2}, \omega_{2}}^{\beta} \mathcal{U}\right)(\varsigma) \\
& +s\left({ }_{0^{+}} \mathscr{J}_{\mathbf{q}_{1}, \omega_{1}}^{\alpha} \mathscr{U}\right)(\varsigma)\left({ }_{0^{+}} \mathscr{J}_{\mathbf{q}_{2}, \omega_{2}}^{\beta} \mathcal{Q}\right)(\varsigma) \\
& \geq\left({ }_{0^{+}} \mathcal{J}_{\mathbf{q}_{1}, \omega_{1}}^{\alpha} \mathbb{Q}^{r} \mathscr{U}^{s}\right)(\varsigma)\left({ }_{0^{+}} \mathcal{J}_{\mathbf{q}_{2}, \omega_{2}}^{\beta} \mathscr{Q}^{s} \mathscr{U}^{r}\right)(\varsigma) \text {, }
\end{aligned}
$$

which implies $\left(A_{5}\right)$. The rest of variants can be derived by adopting the similar strategy and accompanying the selection of parameters in $A M-G M$ inequality as follows:

$$
\begin{aligned}
& \left(A_{6}\right) \rho_{1}=\mathscr{Q}(x) / \mathscr{Q}(y), \rho_{2}=\mathscr{U}(x) / \mathcal{U}(y), \\
& \left(A_{7}\right) \rho_{1}=\mathbb{Q}(x) \mathcal{U}^{2 / r}(y), \rho_{2}=\mathbb{Q}^{2 / s}(x) \mathcal{U}(y), \\
& \left(A_{8}\right) \rho_{1}=Q^{2 / r}(x) \mathscr{Q}(y), \\
& \rho_{2}=\mathscr{U}^{2 / s}(x) \mathscr{U}(y) .
\end{aligned}
$$

Theorem 15. Let $\mathfrak{q} \in(0,1), \omega \geq 0, \mathbb{Q}$ and $\mathcal{U}$ be the positive $\mathfrak{q}, \omega$-integrable functions defined on $[0, \infty)$, and

$$
\begin{gathered}
e=\min _{0 \leq x \leq \varsigma} \frac{\mathbb{Q}(\varsigma)}{\mathscr{U}(\varsigma)}, \\
\mathscr{E}=\max _{0 \leq x \leq \varsigma} \frac{\mathscr{Q}(\varsigma)}{\mathscr{U}(\varsigma)} .
\end{gathered}
$$

Then, the inequalities

$\left(A_{9}\right) \quad 0 \leq\left({ }_{0^{+}} \mathscr{J}_{\mathbf{q}, \omega}^{\alpha} Q^{2}\right)(\varsigma)\left({ }_{0^{+}} \mathscr{I}_{\mathfrak{q}, \omega}^{\alpha} U^{2}\right)(\varsigma) \leq\left((e+\mathscr{E})^{2} / 4 e \mathscr{E}\right)$ $\left({ }_{0^{+}} \mathcal{I}_{\mathbf{q}, \omega}^{\alpha}(\mathbb{Q} \mathscr{U})\right)^{2}(\varsigma)$

$$
\left(A_{10}\right) 0 \leq \sqrt{\left({ }_{0^{+}} \mathscr{J}_{\mathbf{q}, \omega}^{\alpha} Q^{2}\right)(\varsigma)\left({ }_{0^{+}} \mathscr{J}_{\mathbf{q}, \omega}^{\alpha} \mathcal{U}^{2}\right)(\varsigma)}-\left({ }_{0^{+}} \mathscr{J}_{\mathbf{q}, \omega}^{\alpha} Q \mathcal{Q}\right)
$$

$(\varsigma) \leq\left((\sqrt{\mathscr{E}}-\sqrt{e})^{2} / 2 \sqrt{e \mathscr{E}}\right)\left({ }_{0^{+}} \mathscr{J}_{\mathbf{q}, \omega}^{\alpha} Q \mathcal{Q}\right)(\varsigma)$

$\left(A_{11}\right) \quad 0 \leq\left({ }_{0^{+}} \mathscr{J}_{\mathbf{q}, \omega}^{\alpha} \mathbb{Q}^{2}\right)(\varsigma)\left({ }_{0^{+}} \mathscr{J}_{\mathbf{q}, \omega}^{\alpha} \mathscr{U}^{2}\right)(\varsigma)-\left({ }_{0^{+}} \mathscr{J}_{\mathbf{q}, \omega}^{\alpha} \mathbb{Q} \mathcal{U}\right)^{2}$

$(\varsigma) \leq\left((\mathscr{E}-e)^{2} / 4 e \mathscr{E}\right)\left({ }_{0^{+}} \mathscr{J}_{\mathrm{q}, \omega}^{\alpha}(\mathscr{Q} \mathscr{U})\right)^{2}(\varsigma)$

hold for all $\varsigma>0$.

Proof. From (49) and (50), we clearly see that

$$
\left(\frac{\mathscr{Q}(x)}{\mathscr{U}(x)}-e\right)\left(\mathscr{E}-\frac{\mathscr{Q}(x)}{\mathcal{U}(x)}\right) \mathcal{U}^{2}(x) \geq 0(0 \leq x<\varsigma) .
$$

Conducting product on both sides of (51) by $\left(\varsigma-{ }_{\sigma} \Psi_{\mathrm{q}}\right.$ $(x))_{\sigma}^{(\alpha-1)} / \Gamma_{\mathfrak{q}}(\alpha)$ and then performing the $\mathfrak{q}, \omega$-integration with respect to $x$ over $(0, \varsigma)$ yield

$$
\left({ }_{0^{+}} \mathscr{I}_{\mathbf{q}, \omega}^{\alpha} Q^{2}\right)(\varsigma)+e \mathscr{E}\left({ }_{0^{+}} \mathscr{I}_{\mathbf{q}, \omega}^{\alpha} \mathscr{U}^{2}\right)(\varsigma) \leq(e+\mathscr{E})\left({ }_{0^{+}} \mathscr{I}_{\mathbf{q}, \omega}^{\alpha} Q \mathcal{Q} U\right)(\varsigma) .
$$

It follows from $e \mathscr{E}>0$ and

$$
\sqrt{\left({ }_{0^{+}} \mathscr{I}_{\mathbf{q}, \omega}^{\alpha} Q^{2}\right)(\varsigma)}-\sqrt{e^{\mathscr{E}}\left({ }_{0^{+}} \mathcal{I}_{\mathbf{q}, \omega}^{\alpha} \mathscr{U}^{2}\right)(\varsigma)} \geq 0
$$

that

$$
\begin{aligned}
& 2 \sqrt{\left({ }_{0^{+}} \mathcal{J}_{\mathfrak{q}, \omega}^{\alpha} \mathscr{Q}^{2}\right)(\varsigma)} \sqrt{e_{\mathscr{E}}^{\mathscr{E}}\left({ }_{0^{+}} \mathcal{J}_{\mathfrak{q}, \omega}^{\alpha} \mathscr{U}^{2}\right)(\varsigma)} \\
& \quad \leq\left({ }_{0^{+}} \mathcal{J}_{\mathbf{q}, \omega}^{\alpha} \mathscr{Q}^{2}\right)(\varsigma)+(e \mathscr{E})\left({ }_{0^{+}} \mathscr{J}_{\mathfrak{q}, \omega}^{\alpha} \mathscr{U}^{2}\right)(\varsigma) .
\end{aligned}
$$

From (52) and (54), we conclude that

$$
\left({ }_{0^{+}} \mathscr{J}_{\mathbf{q}, \omega}^{\alpha} \mathbb{Q}^{2}\right)(\varsigma)\left({ }_{0^{+}} \mathcal{J}_{\mathbf{q}, \omega}^{\alpha} \mathscr{U}^{2}\right)(\varsigma) \leq \frac{(e+\mathscr{E})^{2}}{4 e \mathscr{E}}\left({ }_{0^{+}} \mathscr{J}_{\mathbf{q}, \omega}^{\alpha}(\mathscr{Q} \mathscr{U})\right)^{2}(\varsigma),
$$

which implies $\left(A_{9}\right)$. By making few changes in $\left(A_{9}\right)$, we can get $\left(A_{10}\right)$ and $\left(A_{11}\right)$.

Theorem 16. Let $\mathfrak{q} \in(0,1), \omega \geq 0$, and $\mathbb{Q}$ and $\mathscr{U}$ be the positive $\mathfrak{q}, \omega$-integrable functions defined on $[0, \infty)$ such that

$$
\begin{gathered}
0<\delta_{1} \leq \mathscr{Q}(x) \leq \delta_{2}<\infty, \\
0<\Delta_{1} \leq \mathscr{U}(x) \leq \Delta_{2}<\infty .
\end{gathered}
$$

Then, the inequalities

$$
\left(A_{12}\right) 0 \leq\left({ }_{0^{+}} \mathcal{J}_{\mathbf{q}, \omega}^{\alpha} Q^{2}\right)(\varsigma)\left({ }_{0^{+}} \mathcal{I}_{\mathbf{q}, \omega}^{\alpha} \mathscr{U}^{2}\right)(\varsigma) \leq\left(\left(\delta_{1} \Delta_{1}+\delta_{2} \Delta_{2}\right)^{2} /\right.
$$
$\left.4 \delta_{1} \Delta_{1} \delta_{2} \Delta_{2}\right)\left({ }_{0^{+}}^{+} \mathscr{J}_{\mathbf{q}, \omega}^{\alpha}(Q \mathcal{Q} U)\right)^{2}(\varsigma)$

$$
\begin{aligned}
& \left(A_{13}\right) 0 \leq \sqrt{\left({ }_{0^{+}} \mathcal{F}_{\mathfrak{q}, \omega}^{\alpha} Q^{2}\right)(\varsigma)\left({ }_{0^{+}} \mathcal{F}_{\mathfrak{q}, \omega}^{\alpha} \mathscr{U}^{2}\right)(\varsigma)}-\left({ }_{0^{+}} \mathcal{F}_{\mathfrak{q}, \omega}^{\alpha} Q \mathcal{Q} \mathcal{U}\right) \\
& (\varsigma) \leq\left(\left(\sqrt{\delta_{2} \Delta_{2}}-\sqrt{\delta_{1} \Delta_{1}}\right)^{2} / 2 \sqrt{\delta_{1} \Delta_{1} \delta_{2} \Delta_{2}}\right)\left({ }_{0^{+}} \mathcal{J}_{\mathfrak{q}, \omega}^{\alpha} \mathbb{Q} \mathcal{U}\right)(\varsigma) \\
& \left(A_{14}\right) 0 \leq\left({ }_{0^{+}} \mathcal{J}_{\mathbf{q}, \omega}^{\alpha} \mathbb{Q}^{2}\right)(\varsigma)\left({ }_{0^{+}} \mathcal{F}_{\mathbf{q}, \omega}^{\alpha} \mathscr{U}^{2}\right)(\varsigma)-\left({ }_{0^{+}} \mathcal{F}_{\mathbf{q}, \omega}^{\alpha} \mathbb{Q} \mathcal{U}\right)^{2}(\varsigma) \\
& \leq\left(\left(\delta_{2} \Delta_{2}-\delta_{1} \Delta_{1}\right)^{2} / 4 \delta_{1} \Delta_{1} \delta_{2} \Delta_{2}\right)\left({ }_{0^{+}} \mathscr{J}_{\mathbf{q}, \omega}^{\alpha}(\mathbb{Q} \mathscr{U})\right)^{2}(\varsigma)\left(A_{14}\right) \\
& \text { hold for all } \varsigma>0 \text {. }
\end{aligned}
$$


Proof. It follows from (56) and (57) that

$$
\frac{\delta_{1}}{\Delta_{2}} \leq \frac{\mathscr{Q}(x)}{\mathcal{U}(x)} \leq \frac{\delta_{2}}{\Delta_{1}}
$$

The proof can be derived by following Theorem 15 .

Theorem 17. Let $r, s>0$ with $r+s=1, \mathfrak{q} \in(0,1), \omega \geq 0, \mathscr{P}, \mathbb{Q}$ and $\mathcal{U}$ be the positive $\mathfrak{q}, \omega$-integrable functions defined on $[0, \infty)$ such that (56) and (57) hold. Then, the inequalities

$\left(A_{15}\right)\left({ }_{0^{+}} \mathscr{J}_{\mathbf{q}, \omega}^{\alpha} \mathscr{P} Q\right)^{s}(\varsigma)\left({ }_{0^{+}} \mathscr{J}_{\mathbf{q}, \omega}^{\alpha}(\mathscr{P} / \mathscr{Q})\right)^{r}(\varsigma) \leq\left(r \delta_{1}+s \delta_{2} /\right.$ $\left.\left(\delta_{1} \delta_{2}\right)^{r}\right)\left({ }_{0^{+}} \mathscr{J}_{\mathbf{q}, \omega}^{\alpha} \mathscr{P}\right)(\varsigma)$

$\left(A_{1 \sigma}\right)\left({ }_{0^{+}} \mathscr{J}_{\mathfrak{q}, \omega}^{\alpha} \mathscr{P} Q\right)^{s}(\varsigma)\left({ }_{0^{+}} \mathscr{J}_{\mathfrak{q}, \omega}^{\alpha} \mathscr{P} \mathcal{U}\right)^{r}(\varsigma) \leq\left(r \delta_{1} \Delta_{1}+s \delta_{2} \Delta_{2} /\right.$ $\left.\left(\delta_{1} \delta_{2}\right)^{r}\left(\Delta_{1} \Delta_{2}\right)^{s}\right)\left({ }_{0^{+}} \mathscr{J}_{\mathbf{q}, \omega}^{\alpha} \mathscr{P} \mathscr{Q} \mathcal{U}\right)^{s}(\varsigma)\left(A_{16}\right)$

hold for all $\varsigma>0$.

Proof. It follows from $\left(s \mathscr{Q}(x)-r \delta_{1}\right)\left(\mathscr{Q}(x)-\delta_{2}\right) \leq 0$ that

$$
s Q^{2}(x)-\left(r \delta_{1}+s \delta_{2}\right) \mathscr{Q}(x)+r \delta_{1} \delta_{2} \leq 0 .
$$

Multiplying both sides of (59) by $\mathscr{P}(x) / \mathscr{Q}(x)$, we have

$$
s \mathscr{P}(x) \mathscr{Q}(x)+r \delta_{1} \delta_{2} \frac{\mathscr{P}(x)}{\mathscr{Q}(x)} \leq\left(r \delta_{1}+s \delta_{2}\right) \mathscr{P}(x)
$$

Taking into account the well-known $A M-G M$ inequality for (60), we get

$$
\begin{aligned}
& \left(\int_{0}^{\varsigma} \frac{\left(\varsigma-{ }_{\sigma} \Psi_{\mathfrak{q}}(x)\right)_{\sigma}^{\alpha-1}}{\Gamma_{\mathfrak{q}}(\alpha)} \mathscr{P}(x) \mathcal{Q}(x) d_{\mathfrak{q}, \omega} x\right)^{s} \\
& \cdot\left(\int_{0}^{\varsigma} \frac{\left(\varsigma-{ }_{\sigma} \Psi_{\mathfrak{q}}(x)\right)_{\sigma}^{\alpha-1}}{\Gamma_{\mathfrak{q}}(\alpha)} \frac{\mathscr{P}(x)}{\mathscr{Q}(x)} d_{\mathfrak{q}, \omega} x\right)^{r} \\
& =\frac{1}{\left(\delta_{1} \delta_{2}\right)^{r}}\left(\int_{0}^{\varsigma} \frac{\left(\varsigma-{ }_{\sigma} \Psi_{\mathfrak{q}}(x)\right)_{\sigma}^{\alpha-1}}{\Gamma_{\mathfrak{q}}(\alpha)} \mathscr{P}(x) \mathscr{Q}(x) d_{\mathfrak{q}, \omega} x\right)^{s} \\
& \cdot\left(\delta_{1} \delta_{2} \int_{0}^{\varsigma} \frac{\left(\varsigma-{ }_{\sigma} \Psi_{\mathfrak{q}}(x)\right)_{\sigma}^{\alpha-1}}{\Gamma_{\mathfrak{q}}(\alpha)} \frac{\mathscr{P}(x)}{Q(x)} d_{\mathfrak{q}, \omega} x\right)^{r} \\
& \leq \frac{1}{\left(\delta_{1} \delta_{2}\right)^{r}}\left(\frac{s}{\Gamma_{\mathfrak{q}}(\alpha)} \int_{0}^{\varsigma}\left(\varsigma-{ }_{\sigma} \Psi_{\mathfrak{q}}(x)\right)_{\sigma}^{\alpha-1} \mathscr{P}(x) \mathscr{Q}(x) d_{\mathfrak{q}, \omega} x\right. \\
& \left.+\frac{r \delta_{1} \delta_{2}}{\Gamma_{\mathfrak{q}}(\alpha)} \int_{0}^{\varsigma}\left(\varsigma-{ }_{\sigma} \Psi_{\mathfrak{q}}(x)\right)_{\sigma}^{\alpha-1} \frac{\mathscr{P}(x)}{\mathscr{Q}(x)} d_{\mathfrak{q}, \omega} x\right) \\
& \leq \frac{r \delta_{1}+s \delta_{2}}{\left(\delta_{1} \delta_{2}\right)^{r}}\left(\frac{1}{\Gamma_{\mathfrak{q}}(\alpha)} \int_{0}^{\varsigma}\left(\varsigma-{ }_{\sigma} \Psi_{\mathfrak{q}}(x)\right)_{\sigma}^{\alpha-1} \mathscr{P}(x) d_{\mathfrak{q}, \omega} x\right),
\end{aligned}
$$

which implies $\left(A_{15}\right)$.
Replacing, respectively, $\mathscr{P}$ and $\mathbb{Q}$ by $\mathscr{P} Q \mathcal{U}$ and $\mathcal{Q} / \mathcal{U}$ in $(61)$ and (56), we attain the required inequality $\left(A_{16}\right)$ :

$$
\begin{aligned}
& \left(\int_{0}^{\varsigma} \frac{\left(\varsigma-{ }_{\sigma} \Psi_{\mathfrak{q}}(x)\right)_{\sigma}^{\alpha-1}}{\Gamma_{\mathfrak{q}}(\alpha)} \mathscr{P}(x) \mathscr{Q}(x) d_{\mathfrak{q}, \omega} x\right)^{s} \\
& \quad \cdot\left(\int_{0}^{\varsigma} \frac{\left(\varsigma-{ }_{\sigma} \Psi_{\mathfrak{q}}(x)\right)_{\sigma}^{\alpha-1}}{\Gamma_{\mathfrak{q}}(\alpha)} \mathscr{P}(x) \mathscr{U}(x) d_{\mathfrak{q}, \omega} x\right)^{r} \\
& \leq \frac{r \delta_{1} \Delta_{1}+s \delta_{2} \Delta_{2}}{\left(\delta_{1} \delta_{2}\right)^{r}\left(\Delta_{1} \Delta_{2}\right)^{s}}\left(\int_{0}^{\varsigma} \frac{\left(\varsigma-{ }_{\sigma} \Psi_{\mathfrak{q}}(x)\right)_{\sigma}^{\alpha-1}}{\Gamma_{\mathfrak{q}}(\alpha)} \mathscr{P}(x) \mathscr{Q}(x) \mathcal{U}(x) d_{\mathfrak{q}, \omega} x\right)^{s} .
\end{aligned}
$$

Theorem 18. Let $\mathfrak{q} \in(0,1), \omega \geq 0$, and $\mathscr{P}, \mathbb{Q}$ and $\mathcal{U}$ be the $\mathfrak{q}, \omega$-integrable functions defined on $[0, \infty)$ such that $\mathscr{P}(x) \geq 0$ and (56) holds. Then, for all $\varsigma>0$, we have

$\left(A_{17}\right) \delta_{1} \delta_{2}\left({ }_{0^{+}} \mathscr{J}_{\mathbf{q}, \omega}^{\alpha} \mathscr{P} \mathscr{U}^{2}\right)(\varsigma)+\Delta_{1} \Delta_{2}\left({ }_{0^{+}} \mathscr{J}_{\mathbf{q}, \omega}^{\alpha} \mathscr{P} Q^{2}\right)(\varsigma) \leq\left(\delta_{1} \Delta_{1}+\right.$ $\left.\delta_{2} \Delta_{2}\right)\left({ }_{0^{+}} \mathscr{J}_{\mathfrak{q}, \omega}^{\alpha} \mathscr{P} \mathscr{Q} \mathscr{U}\right)(\varsigma) \leq\left|\delta_{1} \Delta_{1}+\delta_{2} \Delta_{2}\right|\left[\left({ }_{0^{+}} \mathscr{J}_{\mathfrak{q}, \omega}^{\alpha} \mathscr{P} Q^{2}\right)(\varsigma)+\right.$ $\left.\left({ }_{0^{+}} \mathscr{J}_{\mathfrak{q}, \omega}^{\alpha} \mathscr{P} \mathcal{U}^{2}\right)(\varsigma)\right]\left(A_{17}\right)$

$\left(A_{18}\right) \sqrt{\delta_{1} \delta_{2} / \Delta_{1} \Delta_{2}}\left({ }_{0^{+}} \mathscr{I}_{\mathfrak{q}, \omega}^{\alpha} \mathscr{P} \mathcal{U}^{2}\right)(\varsigma)+\sqrt{\Delta_{1} \Delta_{2} / \delta_{1} \delta_{2}}\left({ }_{0^{+}} \mathscr{I}_{\mathfrak{q}, \omega}^{\alpha}\right.$ $\left.\mathscr{P} Q^{2}\right)(\varsigma) \leq\left(\sqrt{\delta_{1} \Delta_{1} / \delta_{2} \Delta_{2}}+\sqrt{\delta_{2} \Delta_{2} / \delta_{1} \Delta_{1}}\right)\left({ }_{0^{+}} \mathscr{J}_{\mathbf{q}, \omega}^{\alpha} \mathscr{P} \mathscr{Q} \mathcal{U}\right)(\varsigma)\left(A_{18}\right)$

$\left(A_{19}\right)\left({ }_{0^{+}} \mathscr{J}_{\mathfrak{q}, \omega}^{\alpha} \mathscr{P} \mathscr{U}^{2}\right)(\varsigma)\left({ }_{0^{+}} \mathscr{J}_{\mathfrak{q}, \omega}^{\alpha} \mathscr{P} \mathscr{Q}^{2}\right)(\varsigma) \leq\left(\delta_{1} \Delta_{1}+\delta_{2} \Delta_{2} /\right.$ $\left.2 \delta_{1} \Delta_{1} \delta_{2} \Delta_{2}\right)^{2}\left({ }_{0^{+}} \mathscr{J}_{\mathbf{q}, \omega}^{\alpha} \mathscr{P} Q \mathcal{U}\right)(\varsigma)\left(A_{19}\right)$

Proof. We clearly see that

$$
\mathscr{P}(x)\left(\delta_{2} \mathscr{U}(x)-\Delta_{1} \mathscr{Q}(x)\right)\left(\Delta_{2} \mathscr{Q}(x)-\delta_{1} \mathscr{U}(x)\right) \geq 0(x \geq 0) .
$$

Inequality (63) can be written as

$$
\begin{gathered}
\delta_{1} \delta_{2} \mathscr{P}(x) \mathcal{U}^{2}(x)+\Delta_{1} \Delta_{2} \mathscr{P}(x) Q^{2}(x) \\
\leq\left(\delta_{1} \Delta_{1}+\delta_{2} \Delta_{2}\right) \mathscr{P}(x) \mathscr{Q}(x) \mathcal{U}(x) .
\end{gathered}
$$

Conducting product on both sides of (64) by $\left(\varsigma-{ }_{\sigma} \Psi_{\mathfrak{q}}\right.$ $(x))_{\sigma}^{(\alpha-1)} / \Gamma_{\mathfrak{q}}(\alpha)$ and then performing the $\mathfrak{q}, \omega$-integration with respect to $x$ over $(0, \varsigma)$ yield

$$
\begin{aligned}
& \delta_{1} \delta_{2}\left(0_{0^{+}} \mathscr{J}_{\mathfrak{q}, \omega}^{\alpha} \mathscr{P} \mathcal{U}^{2}\right)(\varsigma)+\Delta_{1} \Delta_{2}\left(0_{0^{+}} \mathscr{J}_{\mathfrak{q}, \omega}^{\alpha} \mathscr{P} \mathbb{Q}^{2}\right)(\varsigma) \\
& \leq\left(\delta_{1} \Delta_{1}+\delta_{2} \Delta_{2}\right)\left({ }_{0^{+}} \mathscr{J}_{\mathfrak{q}, \omega}^{\alpha} \mathscr{P} \mathscr{Q} \mathcal{U}\right)(\varsigma) .
\end{aligned}
$$

Also, by Cauchy inequality, we get

$$
\begin{aligned}
& \delta_{1} \delta_{2}\left(0_{0^{+}} \mathscr{J}_{\mathfrak{q}, \omega}^{\alpha} \mathscr{P} \mathscr{U}^{2}\right)(\varsigma)+\Delta_{1} \Delta_{2}\left({ }_{0^{+}} \mathscr{J}_{\mathfrak{q}, \omega}^{\alpha} \mathscr{P} Q^{2}\right)(\varsigma) \\
& \leq\left(\delta_{1} \Delta_{1}+\delta_{2} \Delta_{2}\right)\left({ }_{0^{+}} \mathscr{J}_{\mathfrak{q}, \omega}^{\alpha} \mathscr{P} Q \mathcal{U}\right)(\varsigma) \\
& \leq\left|\delta_{1} \Delta_{1}+\delta_{2} \Delta_{2}\right|\left[\left({ }_{0^{+}} \mathscr{J}_{\mathbf{q}, \omega}^{\alpha} \mathscr{P} \mathbb{Q}^{2}\right)(\varsigma)+\left({ }_{0^{+}} \mathscr{I}_{\mathfrak{q}, \omega}^{\alpha} \mathscr{P} \mathcal{U}^{2}\right)(\varsigma)\right] \text {. }
\end{aligned}
$$

Multiplying both sides of the inequality (66) by $1 /$ $\sqrt{\delta_{1} \delta_{2} \Delta_{1} \Delta_{2}}$, we get $\left(A_{17}\right)$. 
Alternately, it follows from $\delta_{1} \delta_{2} \Delta_{1} \Delta_{2}>0$ and

$$
\left(\sqrt{\delta_{1} \delta_{2}\left(0^{+} \mathcal{I}_{\mathbf{q}, \omega}^{\alpha} \mathscr{P} \mathcal{U}^{2}\right)(\varsigma)}-\sqrt{\Delta_{1} \Delta_{2}\left({ }_{0^{+}} \mathcal{I}_{\mathfrak{q}, \omega}^{\alpha} \mathscr{P} \mathscr{Q}^{2}\right)(\varsigma)}\right)^{2} \geq 0
$$

that

$$
\begin{aligned}
& 2 \sqrt{\delta_{1} \delta_{2}\left({ }_{0^{+}} \mathscr{J}_{\mathbf{q}, \omega}^{\alpha} \mathscr{P} \mathscr{U}^{2}\right)(\varsigma)} \sqrt{\Delta_{1} \Delta_{2}\left({ }_{0^{+}} \mathcal{J}_{\mathbf{q}, \omega}^{\alpha} \mathscr{P} Q^{2}\right)(\varsigma)} \\
& \quad \leq \delta_{1} \delta_{2}\left({ }_{0^{+}} \mathscr{J}_{\mathbf{q}, \omega}^{\alpha} \mathscr{P} \mathscr{U}^{2}\right)(\varsigma)+\Delta_{1} \Delta_{2}\left({ }_{0^{+}} \mathscr{I}_{\mathbf{q}, \omega}^{\alpha} \mathscr{P} \mathscr{Q}^{2}\right)(\varsigma) .
\end{aligned}
$$

From $\left(A_{17}\right)$ and (68), we get

$$
\begin{aligned}
& 4 \delta_{1} \delta_{2} \Delta_{1} \Delta_{2}\left({ }_{0^{+}} \mathcal{J}_{\mathbf{q}, \omega}^{\alpha} \mathscr{P} \mathcal{U}^{2}\right)(\varsigma)\left({ }_{0^{+}} \mathcal{J}_{\mathbf{q}, \omega}^{\alpha} \mathscr{P} \mathscr{Q}^{2}\right)(\varsigma) \\
& \leq\left(\delta_{1} \Delta_{2}+\delta_{2} \Delta_{1}\right)_{0^{+}} \mathscr{J}_{\mathfrak{q}, \omega}^{\alpha} \mathscr{P} \mathcal{U} \mathscr{Q}^{2}(\varsigma),
\end{aligned}
$$

which conclude $\left(A_{19}\right)$. Similarly, we can prove the inequality $\left(A_{18}\right)$.

Theorem 19. Let $\mathfrak{q} \in(0,1), \omega \geq 0$, and $\mathscr{P}, \mathscr{Q}$ and $\mathcal{U}$ be the $\mathfrak{q}, \omega$-integrable functions defined on $[0, \infty)$ such that $\mathscr{P}(x) \geq$ 0 and

$$
\left(\delta_{2} \mathscr{U}(x)-\Delta_{1} \mathscr{Q}(y)\right)\left(\Delta_{2} \mathbb{Q}(y)-\delta_{1} \mathscr{U}(x)\right) \geq 0
$$

for all $x, y>0$. Then, for all $\varsigma>0$, we have

$$
\begin{aligned}
\delta_{1} \delta_{1} & \left({ }_{0^{+}} \mathscr{J}_{\mathbf{q}, \omega}^{\alpha} \mathscr{P}\right)(\varsigma)\left({ }_{0^{+}} \mathscr{J}_{\mathbf{q}, \omega}^{\alpha} \mathscr{P} \mathscr{U}^{2}\right)(\varsigma) \\
& +\Delta_{1} \Delta_{2}\left(0_{0^{+}} \mathscr{J}_{\mathbf{q}, \omega}^{\alpha} \mathscr{P}\right)(\varsigma)\left({ }_{0^{+}} \mathscr{J}_{\mathbf{q}, \omega}^{\alpha} \mathscr{P} \mathscr{Q}^{2}\right)(\varsigma) \\
\leq & \left(\delta_{1} \Delta_{1}+\delta_{2} \Delta_{2}\right)\left({ }_{0^{+}} \mathscr{I}_{\mathbf{q}, \omega}^{\alpha} \mathscr{P} \mathscr{Q}\right)\left(\varsigma\left({ }_{0^{+}} \mathscr{J}_{\mathfrak{q}, \omega}^{\alpha} \mathscr{P} \mathscr{U}\right)(\varsigma)\right),
\end{aligned}
$$

$$
\begin{aligned}
& \delta_{1} \delta_{2}\left({ }_{0^{+}} \mathscr{J}_{\mathbf{q}, \omega}^{\alpha} \mathscr{P} \mathscr{U}\right)^{2}(\varsigma)+\Delta_{1} \Delta_{2}\left(0_{0^{+}} \mathscr{J}_{\mathbf{q}, \omega}^{\alpha} \mathscr{P} Q\right)^{2}(\varsigma) \\
& \leq\left(\delta_{1} \Delta_{1}+\delta_{2} \Delta_{2}\right)\left({ }_{0^{+}} \mathcal{F}_{\mathfrak{q}, \omega}^{\alpha} \mathscr{P}\right)(\varsigma)\left({ }_{0^{+}} \mathscr{J}_{\mathbf{q}, \omega}^{\alpha} \mathscr{P} \mathscr{Q} \mathscr{U}\right)(\varsigma) \\
& \delta_{1} \delta_{2}\left({ }_{0^{+}} \mathscr{J}_{\mathbf{q}, \omega}^{\alpha} \mathscr{P} \mathcal{U}\right)^{2}(\varsigma)+\Delta_{1} \Delta_{2}\left(o_{0^{+}} \mathscr{J}_{\mathbf{q}, \omega}^{\alpha} \mathscr{P} Q\right)^{2}(\varsigma) \\
& \leq\left(\delta_{1} \Delta_{1}+\delta_{2} \Delta_{2}\right)\left({ }_{0^{+}} \mathscr{I}_{\mathfrak{q}, \omega}^{\alpha} \mathscr{P} \mathscr{Q}\right)(\varsigma)\left({ }_{0^{+}} \mathscr{J}_{\mathbf{q}, \omega}^{\alpha} \mathscr{P} \mathscr{Q} \mathscr{U}\right)(\varsigma) \text {. }
\end{aligned}
$$

Proof. Under the given assumption, we have

$$
\mathscr{P}(x) \mathscr{P}(y)\left(\delta_{2} \mathscr{U}(x)-\Delta_{1} \mathscr{Q}(y)\right)\left(\Delta_{2} \mathscr{Q}(y)-\delta_{1} \mathscr{U}(x)\right) \geq 0,
$$

which implies that

$$
\begin{aligned}
& \delta_{1} \delta_{1} \mathscr{P}(x) \mathscr{P}(y) \mathscr{U}^{2}(x)+\Delta_{1} \Delta_{2} \mathscr{P}(x) \mathscr{P}(y) \mathscr{Q}^{2}(y) \\
& \quad \leq \delta_{1} \Delta_{1} \mathscr{P}(x) \mathscr{P}(y) \mathscr{Q}(y) \mathscr{U}(x)+\delta_{2} \Delta_{2} \mathscr{P}(x) \mathscr{P}(y) \mathscr{Q}(y) \mathscr{U}(x) .
\end{aligned}
$$

Conducting product on both sides of (75) by

$$
\frac{\left(\varsigma-{ }_{\sigma} \Psi_{\mathfrak{q}}(x)\right)_{\sigma}^{\alpha-1}\left(\varsigma-{ }_{\sigma} \Psi_{\mathfrak{q}}(y)\right)_{\sigma}^{\alpha-1}}{\Gamma_{\mathfrak{q}}^{2}(\alpha)},
$$

and then performing the $\mathfrak{q}, \omega$-integration with respect to $x$ and $y$ over $(0, \varsigma)$, we get $(71)$.

From (65), (71), and the Cauchy inequality

$$
\begin{aligned}
& \left({ }_{0^{+}} \mathscr{J}_{\mathbf{q}, \omega}^{\alpha} \mathscr{P} Q\right)^{2}(\varsigma) \leq\left({ }_{0^{+}} \mathcal{J}_{\mathbf{q}, \omega}^{\alpha} \mathscr{P}\right)(\varsigma)\left({ }_{0^{+}} \mathscr{J}_{\mathbf{q}, \omega}^{\alpha} \mathscr{P} Q^{2}\right)(\varsigma) \text {, } \\
& \left({ }_{0^{+}} \mathscr{J}_{\mathbf{q}, \omega}^{\alpha} \mathscr{P} \mathscr{U}\right)^{2}(\varsigma) \leq\left({ }_{0^{+}} \mathscr{J}_{\mathbf{q}, \omega}^{\alpha} \mathscr{P}\right)(\varsigma)\left({ }_{0^{+}} \mathscr{I}_{\mathbf{q}, \omega}^{\alpha} \mathscr{P} \mathcal{U}^{2}\right)(\varsigma) \text {, }
\end{aligned}
$$

we get

$$
\begin{aligned}
& \delta_{1} \delta_{2}\left({ }_{0^{+}} \mathscr{J}_{\mathbf{q}, \omega}^{\alpha} \mathscr{P} \mathcal{U}\right)^{2}(\varsigma)+\Delta_{1} \Delta_{2}\left({ }_{0^{+}} \mathscr{J}_{\mathbf{q}, \omega}^{\alpha} \mathscr{P} Q\right)^{2}(\varsigma) \\
& \leq \delta_{1} \delta_{2}\left({ }_{0^{+}} \mathscr{J}_{\mathfrak{q}, \omega}^{\alpha} \mathscr{P}\right)(\varsigma)\left({ }_{0^{+}} \mathscr{I}_{\mathfrak{q}, \omega}^{\alpha} \mathscr{P} \mathscr{Q}^{2}\right)(\varsigma) \\
& +\Delta_{1} \Delta_{2}\left(0_{0^{+}} \mathscr{I}_{\mathrm{q}, \omega}^{\alpha} \mathscr{P}\right)(\varsigma)\left({ }_{0^{+}} \mathscr{J}_{\mathbf{q}, \omega}^{\alpha} \mathscr{P} \mathscr{U}^{2}\right)(\varsigma) \\
& \leq\left(\delta_{1} \Delta_{1}+\delta_{2} \Delta_{2}\right)\left({ }_{0^{+}} \mathscr{J}_{\mathbf{q}, \omega}^{\alpha} \mathscr{P}\right)(\varsigma)\left({ }_{0^{+}} \mathscr{J}_{\mathbf{q}, \omega}^{\alpha} \mathscr{P} \mathscr{Q} \mathscr{U}\right)(\varsigma) \text {, } \\
& \delta_{1} \delta_{2}\left({ }_{0^{+}} \mathscr{J}_{\mathbf{q}, \omega}^{\alpha} \mathscr{P} \mathscr{U}\right)^{2}(\varsigma)+\Delta_{1} \Delta_{2}\left(0_{0^{+}} \mathscr{I}_{\mathbf{q}, \omega}^{\alpha} \mathscr{P} Q\right)^{2}(\varsigma) \\
& \leq\left(\delta_{1} \Delta_{1}+\delta_{2} \Delta_{2}\right)\left({ }_{0^{+}} \mathscr{J}_{\mathfrak{q}, \omega}^{\alpha} \mathscr{P} \mathscr{Q}\right)(\varsigma)\left({ }_{0^{+}} \mathscr{J}_{\mathfrak{q}, \omega}^{\alpha} \mathscr{P} \mathscr{U}\right)(\varsigma),
\end{aligned}
$$

which completes the proof of (72) and (73).

\section{Conclusion}

We have discovered several generalizations for the generalized Čebyšev type inequality via quantum fractional Hahn's integral operator by using the quantum shift operator ${ }_{\sigma} \Psi_{\mathfrak{q}}(\varsigma)=\mathfrak{q} \varsigma+(1-\mathfrak{q}) \sigma\left(\varsigma \in\left[l_{1}, l_{2}\right], \sigma=l_{1}+(\omega /(1-\mathfrak{q})), 0<\right.$ $\mathfrak{q}<1, \omega \geq 0)$, provided some associated variants to show the efficiency of quantum Hahn's integral operator, and compared our obtained results and proposed technique with the previously known results and existing technique. The outcome shows that the proposed plans are extremely important and computationally appealing to deal with several sorts of differential equations. As a future research course of this paper, the new techniques obtained in the present paper can be prolonged to attain analytical solutions of quantum mechanics introduced in different works distributed currently connected with high-dimensional fractional equations.

\section{Data Availability}

The data used to support the findings of this study are available from the corresponding author upon request.

\section{Conflicts of Interest}

The authors declare that there are no conflicts of interest regarding the publication of this paper. 


\section{Authors' Contributions}

All authors contributed equally to writing of this paper. All authors read and approved the final manuscript.

\section{Acknowledgments}

This work was supported by the Natural Science Foundation of China (Grant Nos. 61673169, 11971142, 11871202, 11301127, 11701176, 11626101, and 11601485).

\section{References}

[1] Z.-F. Dai and F.-H. Wen, "Another improved Wei-Yao-Liu nonlinear conjugate gradient method with sufficient descent property," Applied Mathematics and Computation, vol. 218, no. 14, pp. 7421-7430, 2012.

[2] Y. Jiang and J. Ma, "Spectral collocation methods for Volterraintegro differential equations with noncompact kernels," Journal of Computational and Applied Mathematics, vol. 244, pp. 115-124, 2013.

[3] Z.-F. Dai and F.-H. Wen, "Robust CVaR-based portfolio optimization under a genal affine data perturbation uncertainty set," Journal of Computational Analysis and Applications, vol. 16, no. 1, pp. 93-103, 2014.

[4] C. Huang, S. Guo, and L. Liu, "Boundedness on Morrey space for Toeplitz type operator associated to singular integral operator with variable Calderón-Zygmund kernel," Journal of Mathematical Inequalities, vol. 8, no. 3, pp. 453-464, 2014.

[5] W.-J. Zhou and F. Wang, "A PRP-based residual method for large-scale monotone nonlinear equations," Applied Mathematics and Computation, vol. 261, pp. 1-7, 2015.

[6] X. Fang, Y. Deng, and J. Li, "Plasmon resonance and heat generation in nanostructures," Mathematical Methods in the Applied Sciences, vol. 38, no. 18, pp. 4663-4672, 2015.

[7] J. Li, G. Sun, and R. Zhang, "The numerical solution of scattering by infinite rough interfaces based on the integral equation method," Computers \& Mathematics with Applications, vol. 71, no. 7, pp. 1491-1502, 2016.

[8] L. Duan and C. Huang, "Existence and global attractivity of almost periodic solutions for a delayed differential neoclassical growth model," Mathematical Methods in the Applied Sciences, vol. 40, no. 3, pp. 814-822, 2017.

[9] W. Wang, "On A-stable one-leg methods for solving nonlinear Volterra functional differential equations," Applied Mathematics and Computation, vol. 314, pp. 380-390, 2017.

[10] Y. Tan, C. Huang, B. Sun, and T. Wang, "Dynamics of a class of delayed reaction-diffusion systems with Neumann boundary condition," Journal of Mathematical Analysis and Applications, vol. 458, no. 2, pp. 1115-1130, 2018.

[11] A. Tassaddiq, G. Rahman, K. S. Nisar, and M. Samraiz, "Certain fractional conformable inequalities for the weighted and the extended Chebyshev functionals," Advances in Difference Equations, vol. 2020, no. 1, 2020.

[12] Z. Dahmani, O. Mechouar, and S. Brahami, "Certain inequalities related to the Chebyshev's functional involving a Riemann-Liouville operator," Bulletin of Mathematical Analysis and Applications, vol. 3, no. 4, pp. 38-44, 2011.

[13] Z. Dahmani, "New inequalities in fractional integrals," International Journal of Nonlinear Science, vol. 9, no. 4, pp. 493497, 2010.
[14] J. Wang, X. Chen, and L. Huang, "The number and stability of limit cycles for planar piecewise linear systems of node-saddle type," Journal of Mathematical Analysis and Applications, vol. 469, no. 1, pp. 405-427, 2019.

[15] C. Huang, H. Zhang, and L. Huang, "Almost periodicity analysis for a delayed Nicholson's blowflies model with nonlinear density-dependent mortality term," Communications on Pure \& Applied Analysis, vol. 18, no. 6, pp. 3337-3349, 2019.

[16] W. Zhou, "On the convergence of the modified LevenbergMarquardt method with a nonmonotone second order Armijo type line search," Journal of Computational and Applied Mathematics, vol. 239, pp. 152-161, 2013.

[17] W. Zhou and X. Chen, "On the convergence of a modified regularized Newton method for convex optimization with singular solutions," Journal of Computational and Applied Mathematics, vol. 239, pp. 179-188, 2013.

[18] L. Zhang and S. Jian, "Further studies on the Wei-Yao-Liu nonlinear conjugate gradient method," Applied Mathematics and Computation, vol. 219, no. 14, pp. 7616-7621, 2013.

[19] X.-F. Li, G.-J. Tang, and B.-Q. Tang, "Stress field around a strike-slip fault in orthotropic elastic layers via a hypersingular integral equation," Computers \& Mathematics with Applications, vol. 66, no. 11, pp. 2317-2326, 2013.

[20] G. Qin, C. Huang, Y. Xie, and F. Wen, "Asymptotic behavior for third-order quasi-linear differential equations," Advances in Difference Equations, vol. 2013, no. 1, 2013.

[21] M. E. Samei, "Existence of solutions for a system of singular sum fractional $q$-differential equations via quantum calculus," Advances in Difference Equation, vol. 2020, no. 1, 2020.

[22] M. E. Samei, G. K. Ranjbar, and V. Hedayati, "Existence of solutions for equations and inclusions of multiterm fractional $q$-integro-differential with nonseparated and initial boundary conditions," Journal of Inequalities and Applications, vol. 2019, no. 1, 2019.

[23] W. Hahn, "Über Orthogonalpolynome, die q-Differenzengleichungen genügen," Mathematische Nachrichten, vol. 2, no. 1-2, pp. 4-34, 1949.

[24] F. H. Jackson, "q - Difference Equations," American Journal of Mathematics, vol. 32, no. 4, pp. 305-314, 1910.

[25] T. Brikshavana and T. Sitthiwirattham, "On fractional Hahn calculus," Advances in Difference Equations, vol. 2017, no. 1, 2017.

[26] R. S. Costas-Santos and F. Marcellán, "Second structure relation for $q$-semiclassical polynomials of the Hahn Tableau," Journal of Mathematical Analysis and Applications, vol. 329, no. 1, pp. 206-228, 2007.

[27] K. H. Kwon, D. W. Lee, S. B. Park, and B. H. Yoo, "Hahn Class Orthogonal Polynomials," Kyungpook Mathematical Journal, vol. 38, no. 2, pp. 259-281, 1998.

[28] N. Patanarapeelert and T. Sitthiwirattham, "Existence results for fractional Hahn difference and fractional Hahn integral boundary value problems," Discrete Dynamics in Nature and Society, vol. 2017, Article ID 7895186, 2017.

[29] J. Tariboon, S. K. Ntouyas, and P. Agarwal, "New concepts of fractional quantum calculus and applications to impulsive fractional q-difference equations," Advances in Difference Equations, vol. 2015, no. 1, 2015.

[30] J. Tariboon, S. K. Ntouyas, and W. Sudsutad, "New concepts of Hahn calculus and impulsive Hahn difference equations," Advances in Difference Equations, vol. 2016, no. 1, 2016. 
[31] Y. Wang, Y. Liu, and C. Hou, "New concepts of fractional Hahn's q, $\omega$-derivative of Riemann-Liouville type and Caputo type and applications," Advances in Difference Equations, vol. 2018, no. 1, 2018.

[32] A. A. Kilbas, H. M. Srivastava, and J. J. Trujillo, Theory and Applications of Fractional Differential Equations, Elsevier Science B.V, Amsterdam, 2006.

[33] I. Podlubny, Fractional Differential Equations, Academic Press, San Diego, 1999.

[34] M. A. Latif, S. Rashid, S. S. Dragomir, and Y.-M. Chu, "Hermite-Hadamard type inequalities for co-ordinated convex and qausi-convex functions and their applications," Journal of Inequalities and Applications, vol. 2019, no. 1, 2019.

[35] M. U. Awan, N. Akhtar, S. Iftikhar, M. A. Noor, and Y.-M. Chu, "New Hermite-Hadamard type inequalities for n-polynomial harmonically convex functions," Journal of Inequalities and Applications, vol. 2020, no. 1, 2020.

[36] M. A. Khan, N. Mohammad, E. R. Nwaeze, and Y.-M. Chu, "Quantum Hermite-Hadamard inequality by means of a Green function," Advances in Difference Equations, vol. 2020, no. $1,2020$.

[37] A. Iqbal, M. Adil Khan, S. Ullah, and Y.-M. Chu, "Some new Hermite-Hadamard-type inequalities associated with conformable fractional integrals and their applications," Journal of Function Spaces, vol. 2020, Article ID 9845407, 2020.

[38] M. U. Awan, S. Talib, Y.-M. Chu, M. A. Noor, and K. I. Noor, "Some new refinements of Hermite-Hadamard-type inequalities involving $\psi_{k}$-Riemann-Liouville fractional integrals and applications," Mathematical Problems in Engineering, vol. 2020, Article ID 3051920, 2020.

[39] I. Abbas Baloch and Y.-M. Chu, "Petrović-type inequalities for harmonic $h$-convex functions," Journal of Function Spaces, vol. 2020, Article ID 3075390, 2020.

[40] S. Rashid, F. Jarad, H. Kalsoom, and Y.-M. Chu, "On PólyaSzegö and Čebyšev type inequalities via generalized $k$-fractional integrals," Advances in Difference Equations, vol. 2020, no. $1,2020$.

[41] S. Rashid, M. A. Noor, K. I. Noor, and Y.-M. Chu, "Ostrowski type inequalities in the sense of generalized $\mathscr{K}$-fractional integral operator for exponentially convex functions," AIMS Mathematics, vol. 5, no. 3, pp. 2629-2645, 2020.

[42] S. Rashid, F. Jarad, and Y.-M. Chu, "A Note on reverse Minkowski inequality via generalized proportional fractional integral operator with respect to another function," Mathematical Problems in Engineering, vol. 2020, Article ID 7630260, 2020.

[43] S. Khan, M. A. Khan, and Y.-. M. Chu, "Converses of the Jensen inequality derived from the Green functions with applications in information theory," Mathematical Methods in the Applied Sciences, vol. 43, no. 5, pp. 2577-2587, 2020.

[44] M. Adil Khan, J. Pečarić, and Y.-M. Chu, "Refinements of Jensen's and McShane's inequalities with applications," AIMS Mathematics, vol. 5, no. 5, pp. 4931-4945, 2020.

[45] T.-H. Zhao, L. Shi, and Y.-M. Chu, "Convexity and concavity of the modified Bessel functions of the first kind with respect to Hölder means," Revista de la Real Academia de Ciencias Exactas, Físicas y Naturales. Serie A. Matemáticas, vol. 114, no. 2, 2020.

[46] M.-K. Wang, M.-Y. Hong, Y.-F. Xu, Z.-H. Shen, and Y. Chu, "Inequalities for generalized trigonometric and hyperbolic functions with one parameter," Journal of Mathematical Inequalities, vol. 14, no. 1, pp. 1-21, 2020.
[47] Y.-M. Chu, M. A. Khan, T. Ali, and S. S. Dragomir, "Inequalities for $\alpha$-fractional differentiable functions," Journal of Inequalities and Applications, vol. 2017, no. 1, 2017.

[48] S.-S. Zhou, S. Rashid, F. Jarad, H. Kalsoom, and Y.-M. Chu, "New estimates considering the generalized proportional Hadamard fractional integral operators," Advances in Difference Equations, vol. 2020, no. 1, 2020.

[49] Y. Khurshid, M. A. Khan, and Y.-M. Chu, "Conformable fractional integral inequalities for $G G$ - and $G A$-convex functions," AIMS Mathematics, vol. 5, no. 5, pp. 5012-5030, 2020.

[50] S. Rafeeq, H. Kalsoom, S. Hussain, S. Rashid, and Y.-M. Chu, "Delay dynamic double integral inequalities on time scales with applications," Advances in Difference Equations, vol. 2020, no. 1, 2020.

[51] S. Rashid, İ. İșcan, D. Baleanu, and Y.-M. Chu, "Generation of new fractional inequalities via $n$ polynomials $s$-type convexity with applications," Advances in Difference Equations, vol. 2020, 2020.

[52] M.-K. Wang, H.-H. Chu, and Y.-M. Chu, "Precise bounds for the weighted Hölder mean of the complete $p$-elliptic integrals," Journal of Mathematical Analysis and Applications, vol. 480, no. 2, article 123388, 2019.

[53] M.-K. Wang, Z.-Y. He, and Y.-M. Chu, "Sharp power mean inequalities for the generalized elliptic integral of the first kind," Computational Methods and Function Theory, vol. 20, no. 1, pp. 111-124, 2020.

[54] T.-H. Zhao, M.-K. Wang, and Y.-M. Chu, "A sharp double inequality involving generalized complete elliptic integral of the first kind," AIMS Mathematics, vol. 5, no. 5, pp. 45124528, 2020.

[55] M.-K. Wang, H.-H. Chu, Y.-M. Li, and Y.-M. Chu, "Answers to three conjectures on convexity of three functions involving complete elliptic integrals of the first kind," Applicable Analysis and Discrete Mathematics, vol. 14, pp. 255-271, 2020.

[56] W.-M. Qian, Z.-Y. He, and Y.-M. Chu, "Approximation for the complete elliptic integral of the first kind," Revista de la Real Academia de Ciencias Exactas, Físicas y Naturales. Serie A. Matemáticas, vol. 114, no. 2, 2020.

[57] Z.-H. Yang, W.-M. Qian, W. Zhang, and Y.-M. Chu, "Notes on the complete elliptic integral of the first kind," Mathematical Inequalities \& Applications, vol. 23, no. 1, pp. 77-93, 2020.

[58] S. Z. Ullah, M. Adil Khan, and Y.-M. Chu, "A note on generalized convex functions," Journal of Inequalities and Applications, vol. 2019, no. 1, 2019.

[59] M. U. Awan, N. Akhtar, A. Kashuri, M. A. Noor, and Y.M. Chu, " $2 D$ approximately reciprocal $\rho$-convex functions and associated integral inequalities," AIMS Mathematics, vol. 5, no. 5, pp. 4662-4680, 2020.

[60] S. Rashid, R. Ashraf, M. A. Noor, K. I. Noor, and Y.-M. Chu, "New weighted generalizations for differentiable exponentially convex mapping with application," AIMS Mathematics, vol. 5, no. 4, pp. 3525-3546, 2020.

[61] W.-M. Qian, Y.-Y. Yang, H.-W. Zhang, and Y.-M. Chu, "Optimal two-parameter geometric and arithmetic mean bounds for the Sándor-Yang mean," Journal of Inequalities and Applications, vol. 2019, no. 1, 2019.

[62] W.-M. Qian, Z.-Y. He, H.-W. Zhang, and Y.-M. Chu, "Sharp bounds for Neuman means in terms of two-parameter contraharmonic and arithmetic mean," Journal of Inequalities and Applications, vol. 2019, no. 1, 2019. 
[63] B. Wang, C.-L. Luo, S.-H. Li, and Y.-M. Chu, "Sharp oneparameter geometric and quadratic means bounds for the Sándor-Yang means," Revista de la Real Academia de Ciencias Exactas, Físicas y Naturales. Serie A. Matemáticas, vol. 114, no. $1,2020$.

[64] C. Huang, Z. Yang, T. Yi, and X. Zou, "On the basins of attraction for a class of delay differential equations with nonmonotone bistable nonlinearities," Journal of Differential Equations, vol. 256, no. 7, pp. 2101-2114, 2014.

[65] Y.-C. Liu and J. Wu, "Fixed point theorems in piecewise continuous function spaces and applications to some nonlinear problems," Mathematical Methods in the Applied Sciences, vol. 37, no. 4, pp. 508-517, 2014.

[66] W.-S. Tang and Y.-J. Sun, "Construction of Runge-Kutta type methods for solving ordinary differential equations," Applied Mathematics and Computation, vol. 234, pp. 179-191, 2014.

[67] D.-X. Xie and J. Li, "A new analysis of electrostatic free energy minimization and Poisson-Boltzmann equation for protein in ionic solvent," Nonlinear Analysis: Real World Applications, vol. 21, pp. 185-196, 2015.

[68] X.-S. Zhou, "Weighted sharp function estimate and boundedness for commutator associated with singular integral operator satisfying a variant of Hörmander's condition," Journal of Mathematical Inequalities, vol. 9, no. 2, pp. 587-596, 2015.

[69] Z. Dai, X. Chen, and F. Wen, “A modified Perry's conjugate gradient method-based derivative-free method for solving large-scale nonlinear monotone equations," Applied Mathematics and Computation, vol. 270, pp. 378-386, 2015.

[70] Z. Dai, "Comments on a new class of nonlinear conjugate gradient coefficients with global convergence properties," Applied Mathematics and Computation, vol. 276, pp. 297-300, 2016.

[71] Z.-F. Dai, D.-H. Li, and F.-H. Wen, "Worse-case conditional value-at-risk for asymmetrically distributed asset scenarios returns," Journal of Computational Analysis \& Applications, vol. 20, no. 2, pp. 237-251, 2016.

[72] Y. Tan and K. Jing, "Existence and global exponential stability of almost periodic solution for delayed competitive neural networks with discontinuous activations," Mathematical Methods in the Applied Sciences, vol. 39, no. 11, pp. 2821-2839, 2016.

[73] L. Duan, L. Huang, Z. Guo, and X. Fang, "Periodic attractor for reaction-diffusion high-order Hopfield neural networks with time-varying delays," Computers \& Mathematics with Applications, vol. 73, no. 2, pp. 233-245, 2017.

[74] W. Wang and Y. Chen, "Fast numerical valuation of options with jump under Merton's model," Journal of Computational and Applied Mathematics, vol. 318, pp. 79-92, 2017.

[75] C. Huang and L. Liu, "Boundedness of multilinear singular integral operator with non-smooth kernels and mean oscillation," Quaestiones Mathematicae, vol. 40, no. 3, pp. 295-312, 2017.

[76] S. Asawasamrit, C. Sudprasert, S. K. Ntouyas, and J. Tariboon, "Some results on quantum Hahn integral inequalities," Journal of Inequalities and Applications, vol. 2019, no. 1, 2019.

[77] Z. Dahmani, A. Khameli, and K. Freha, "Some RL-integral inequalities for the weighted and the extended Chebyshev functionals," Konuralp Journal of Mathematics, vol. 5, no. 1, pp. 43-48, 2017.

[78] N. Elezović, L. J. Marangunić, and J. Pečarić, "Some improvements of Grüss type inequality," Journal of Mathematical Inequalities, vol. 1, no. 3, pp. 425-436, 2007. 